\title{
Aperçus d'une évolution technique et rituelle en Basse-Birmanie : de la batellerie à la pêche littorale et inter-1̂lienne
}

Glimpses of a technical and ritual evolution in Lower-Burma: from inland water transport to littoral inter-island fishing

Aspectos de una evolución técnica y ritual en Baja Birmania : del barcaje a la pesca litoral e inter-isleña

Luca Gansser et Jacques Ivanoff

\section{OpenEdition}

Journals

Édition électronique

URL : https://journals.openedition.org/tc/299

DOI : $10.4000 /$ tc. 299

ISSN : 1952-420X

\section{Éditeur}

Éditions de l'EHESS

\section{Édition imprimée}

Date de publication : 1 janvier 2001

Pagination : 329-372

ISSN : 0248-6016

\section{Référence électronique}

Luca Gansser et Jacques Ivanoff, « Aperçus d'une évolution technique et rituelle en Basse-Birmanie : de la batellerie à la pêche littorale et inter-îlienne », Techniques \& Culture [En ligne], 35-36 | 2001, mis en ligne le 10 septembre 2012, consulté le 29 septembre 2022. URL : http://journals.openedition.org/tc/ 299 ; DOI : https://doi.org/10.4000/tc.299

Ce document a été généré automatiquement le 29 septembre 2022.

Tous droits réservés 


\title{
Aperçus d'une évolution technique et rituelle en Basse-Birmanie : de la batellerie à la pêche littorale et inter-1̂lienne
}

\author{
Glimpses of a technical and ritual evolution in Lower-Burma: from inland water \\ transport to littoral inter-island fishing \\ Aspectos de una evolución técnica y ritual en Baja Birmania : del barcaje a la \\ pesca litoral e inter-isleña
}

Luca Gansser et Jacques Ivanoff

\section{NOTE DE L'AUTEUR}

Les quatre voyages effectués dans le cadre du MAP ont permis de compléter les données sur les Moken, nomades marins, mais aussi de découvrir et répertorier les différents bateaux de l'archipel et de son littoral. Les courtes enquêtes et la difficulté de faire des haltes prolongées ne nous autorisent qu'une vue partielle de la situation maritime. Nous avons observé surtout des bateaux en mer mais aussi quelques chantiers navals, notamment à Mergui d'où nous tirons la plupart de nos informations sur la construction à propos des navires non monoxyles.

Nous remercions ici F. Robinne pour ses informations et son autorisation de reproduire certains de ses dessins, B. Brac de La Perrière qui nous a aidé à chercher les mots birmans dans le dictionnaire et dont la grande connaissance de la Birmanie nous a permis de nous familiariser avec le domaine des esprits, des génies et de leurs cultes; et bien sûr nous exprimons notre reconnaissance à $D$. Bernot qui a bien voulu nous faire l'honneur de se pencher sur ses carnets de terrain et chercher dans les dictionnaires les étymologies des termes utilisés dans la construction 
navale, ce qui nous a permis de confirmer la plupart de nos hypothèses. Quand nous ne présentons pas les transcriptions locales, nous utilisons les renseignements de ces spécialistes.

La technologie navale des Birmans a toujours été déconsidérée ; les préjugés culturels véhiculés par des "vrais marins » (c'est-à-dire les Occidentaux) se heurtaient à la soidisant suprématie austronésienne des eaux de l'Asie du Sud-Est: «Les Birmans sont mauvais marins et ils ne semblent pratiquer que la navigation de rivière ou en eaux calmes. » (Poujade 1946 : 277) (fig. 1).

2 L'expression de "littoral oublié des Birmans $»^{2}$ (pour reprendre la formulation de F. Robinne 1983) renvoie à l'idée de refus d'une appropriation technique de l'environnement, autour duquel une partie de l'identité birmane s'est constituée et qui est devenu une «logique sociale». Mais cette appropriation limitée ${ }^{3}$ de la mer répondait à des impératifs politiques, économiques et culturels. Les Birmans n'ont colonisé et exploité leur mer que très récemment, les Malais, les Anglais, les Thaïlandais, les Japonais et dernièrement les Indiens s'en occupant, car dans les pays voisins, l'ouverture sur le monde et sur l'économie de marché a permis l'exploitation de la mer, ce qui a donné naissance à un groupe social de marins-pêcheurs, très souvent placé en bas de l'échelle sociale. Aujourd'hui, les marins birmans se sont cependant remarquablement adaptés à leur nouvel environnement, qu'ils apprécient et exploitent de manière manifestement réfléchie et ritualisée, en accord avec leur situation économique. Le littoral est donc maintenant accepté ${ }^{4}$. Quoi qu'il en soit, les embarcations birmanes ont connu une évolution constante au cours des siècles, descendant les fleuves, après être sorties des lacs pour caboter sur les milliers de kilomètres du littoral et des embouchures des fleuves souvent agitées (l'Irrawady particulièrement).

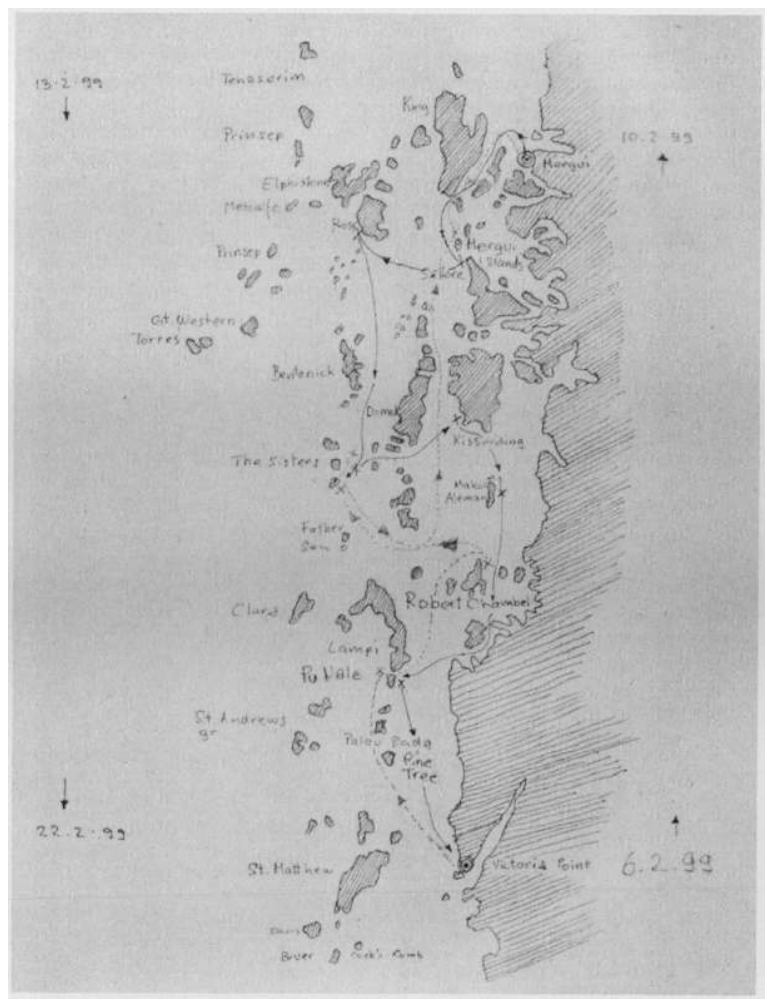

Figure 1. Carte de l'archipel Mergui (L. Gansser) 
L'absence d'études contemporaines concernant les 800 îles de l'archipel Mergui et le littoral du Ténasserim ne permet pas de comparer en matière de technologie navale, ni même de remplir les vides de la recherche générale en ce domaine ${ }^{5}$. Les lacunes ne peuvent donc être comblées que par des hypothèses élaborées à partir de nos enquêtes, de souvenirs d'anciens, complétés de quelques lectures fragmentaires. L'archipel semble aujourd'hui déserté; pourtant, il fut autrefois fréquenté, surtout le port de Mergui (photo 1) qui commandait les îles et le passage que le fleuve Ténasserim offrait aux voyageurs et commerçants se rendant au Siam et plus loin encore ${ }^{6}$. Nous sommes ici dans une zone de contact entre deux mondes, le monde austronésien et le monde indo-birman.

\section{Questions de classification}

Les bateaux du Ténasserim sont localement appelés loñ hlè (fig. 2), bateaux de pêche monoxyles aux bordés en planche ${ }^{7}$ (photos 2 et 3). Le terme loñ (ou loñ, laung ou laung), selon la plupart des auteurs, désigne d'abord les pirogues monoxyles sans bordés, mais aussi les bateaux munis de bordés en planches ${ }^{8}$. Le terme hlè est un terme générique pour «bateau » et recouvre en Basse-Birmanie trois significations : "bateau de mer ", «élever les bordés » et "inclure des planches ». La contradiction apparente entre les définitions du mot loñ (monoxyle ou bateau fait de planches par exemple) disparaît quand on sait que la classification birmane tient compte de la forme plus que de la fonction, de l'usage ou de la structure.

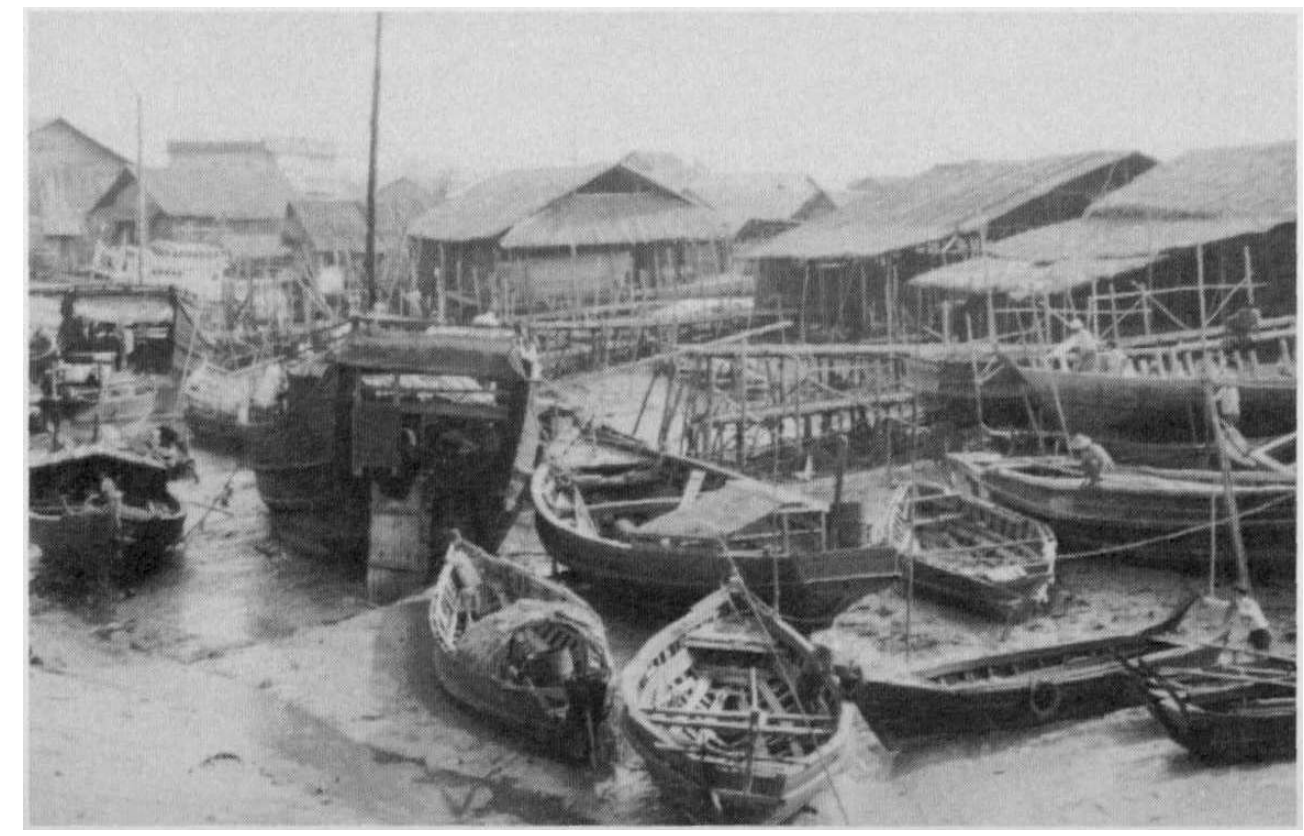

Photo 1. Le vieux port de Mergui (cl. MAP, 1998) 


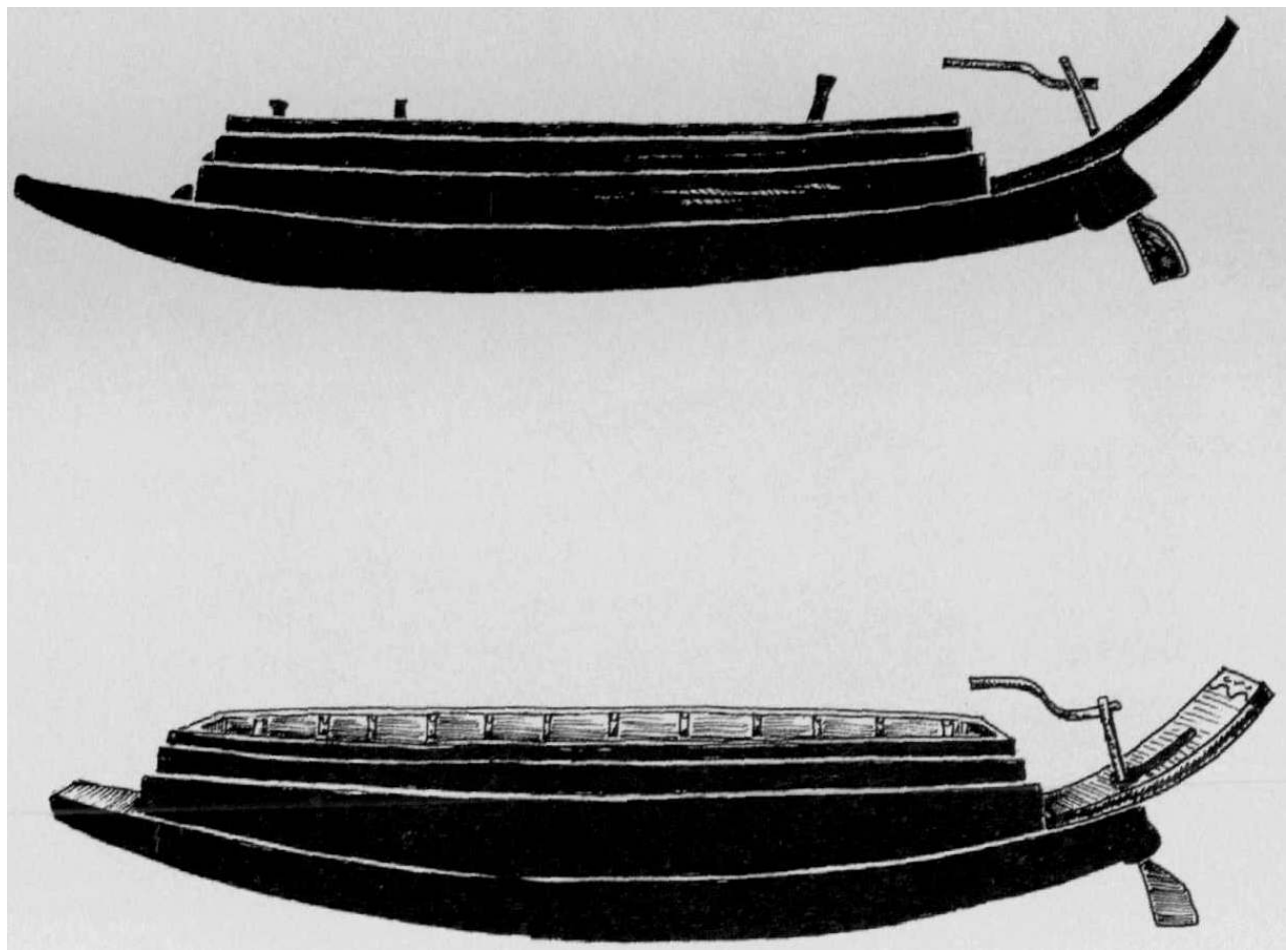

Figure 2. Le loñ blè : la coque monoxyle et trois planches formant bordé (L. Gansser)

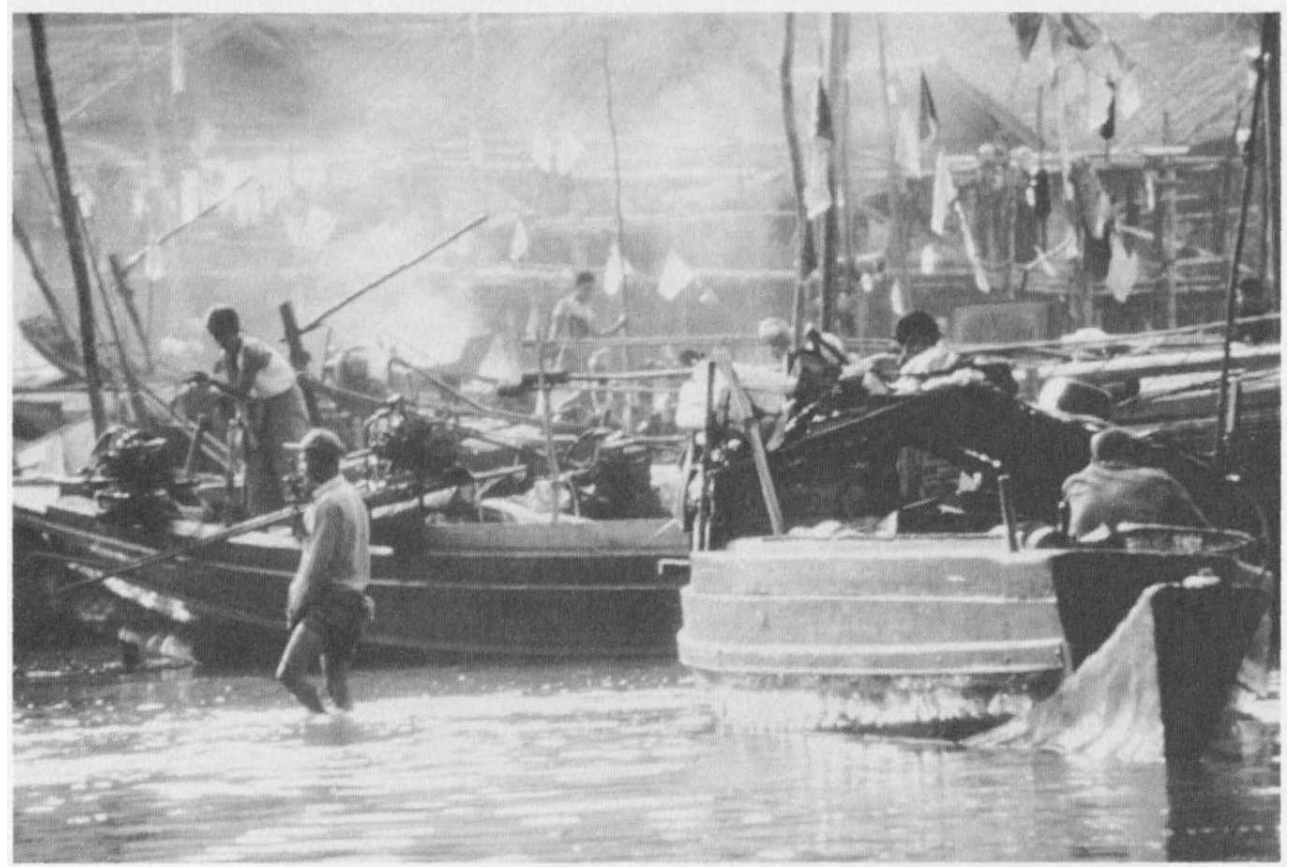




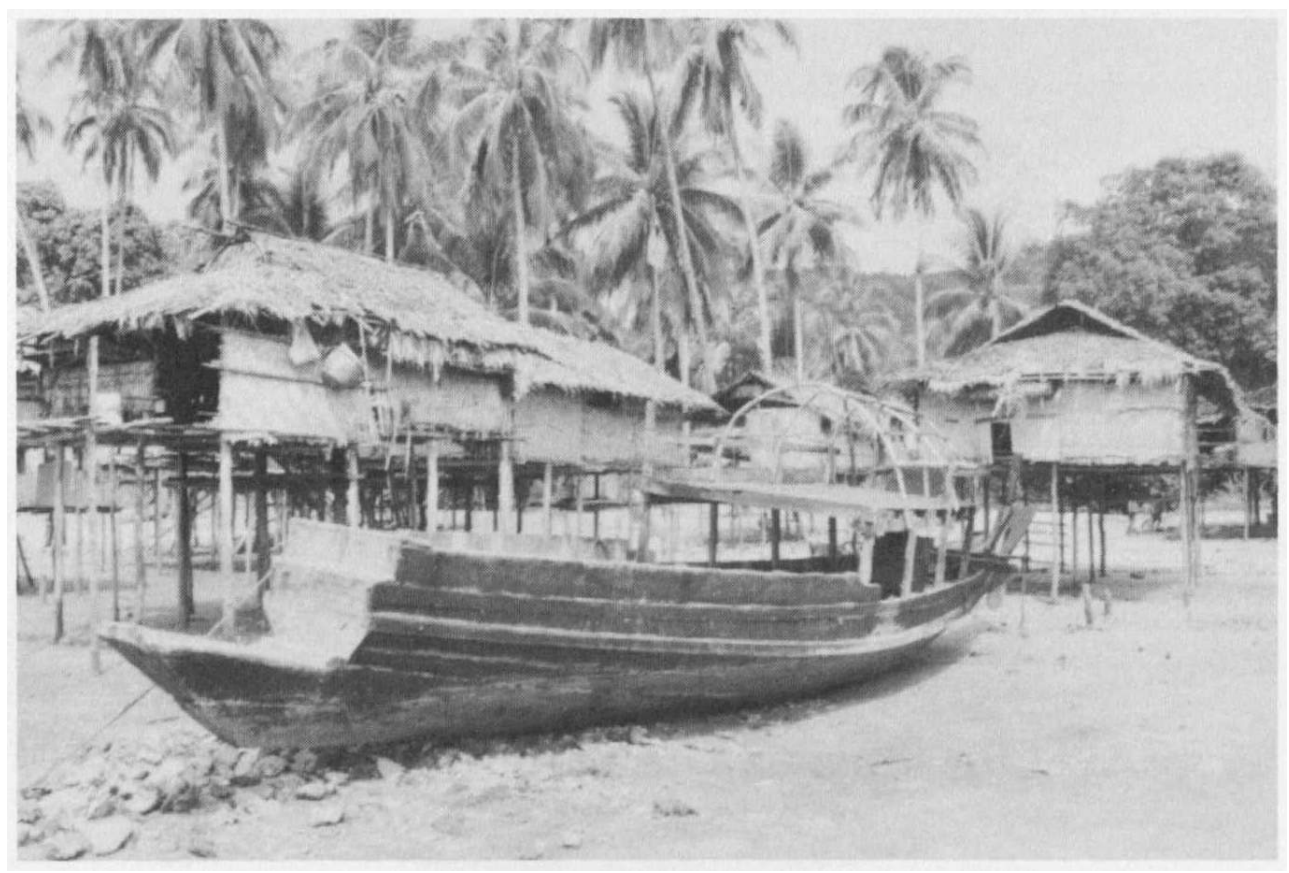

Photos 2 et 3. Le loñ blè en mer et en réparation (cl. MAP, 2000)

La terminologie navale birmane n'est pas suffisamment développée pour permettre un classement adéquat des embarcations. C'est pourquoi tous les auteurs ont fait valoir des classifications assez fantaisistes (Charney 1997). Le dictionnaire Judson (1953: 927), toujours une valeur sûre dans les eaux mouvementées de l'étymologie et de la classification, répartit les bateaux non pas en fonction du type de coque monoxyle ou en planches, mais bien plus d'après la forme (il est vrai souvent dérivée d'un monoxyle) (figs 3 et 4). Pour ce dictionnaire, loñ est un long et mince bateau, le loñ ku (que nous transcrivons loñ ko) un long bateau dont le bordé est composé d'une seule planche ${ }^{9}$, et le loñ zat désigne le même bateau surélevé par un bordé de plusieurs planches. Le terme ko (ou $k u$, ou go, gou), qui suit le terme pein (ou pin), désigne lui-même un corps, une masse, et par conséquent on l'a assimilé au monoxyle. On peut aussi dire que le terme pein go, ou pin ko (fig. 5), est l'association de deux mots, "être compact, masse » et «tarir» ou «ternir ». Ce terme de ternir est peut être simplement une référence à la méthode de protection de la coque au goudron, générale en Birmanie, pays du pétrole. C'est peut-être une des raisons pour lesquelles presque tous les loñ blè sont noirs (photos 2 et 3 ).

6 Enfin le terme loñ blè lui-même, selon le dictionnaire français-birman de D. Bernot (1979) est un canoë -en fait une coque allongée en forme de canoë - ce qui rejoint la définition proposée par Judson. C'est donc bien la forme qui détermine la classification, et le loñ blè est bien le successeur de tous les bateaux fluviaux aux formes allongées, notamment monoxyles, mais pas seulement. La forme allongée rappelle également celle, effilée, des pirogues de course dont nous verrons qu'elles eurent une influence sur le développement des bateaux birmans.

7 Enfin, remarquons, d'un point de vue linguistique encore, que les Birmans sont ouverts et qu'ils intègrent dans leur technique des influences extérieures sans aucun complexe et ne se sentent pas obligés de se référer à une tradition unique et invraisemblable de leurs ancêtres. Il existe ainsi une embarcation, appelée bau'tu, dont le nom serait, selon les dictionnaires (Myanmar-English Dictionnary 1998: 326, par exemple), d'influence 
française ( $c f$. «bateau ») ; cela est possible car les Français ont longtemps fréquenté le littoral birman pour réparer leurs navires.

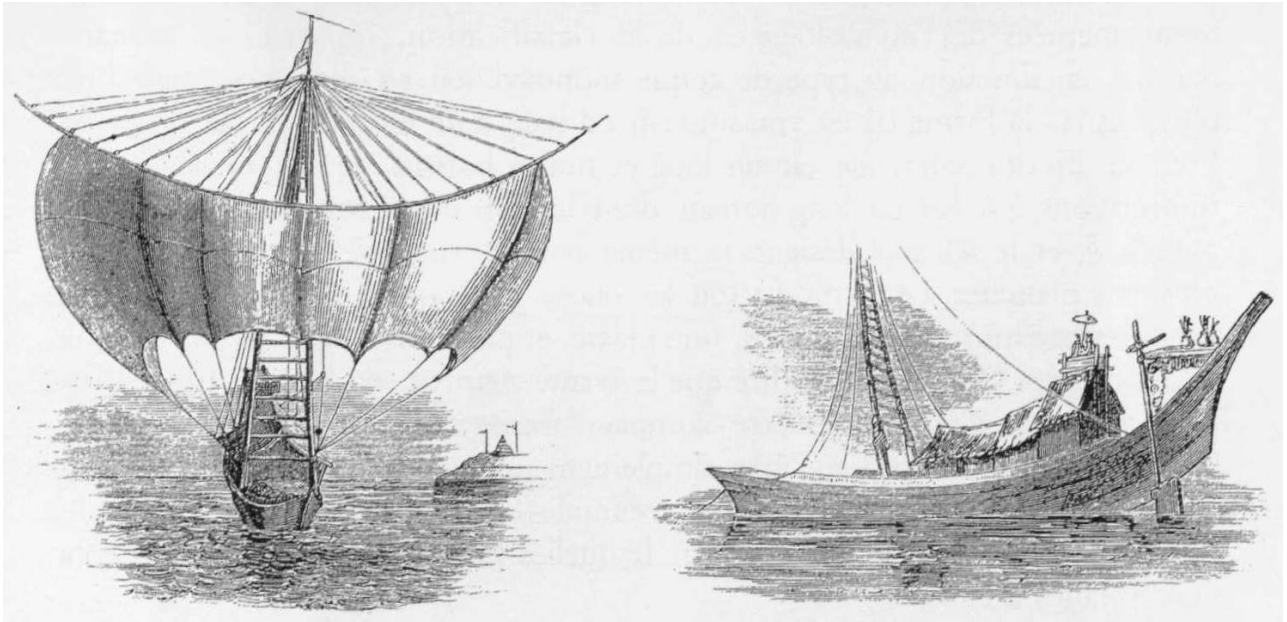

Figures 3 et 4 . Un bateau à voile sur l'Irrawady et un bateau de commerce (Yule $1860: 258-259$ )

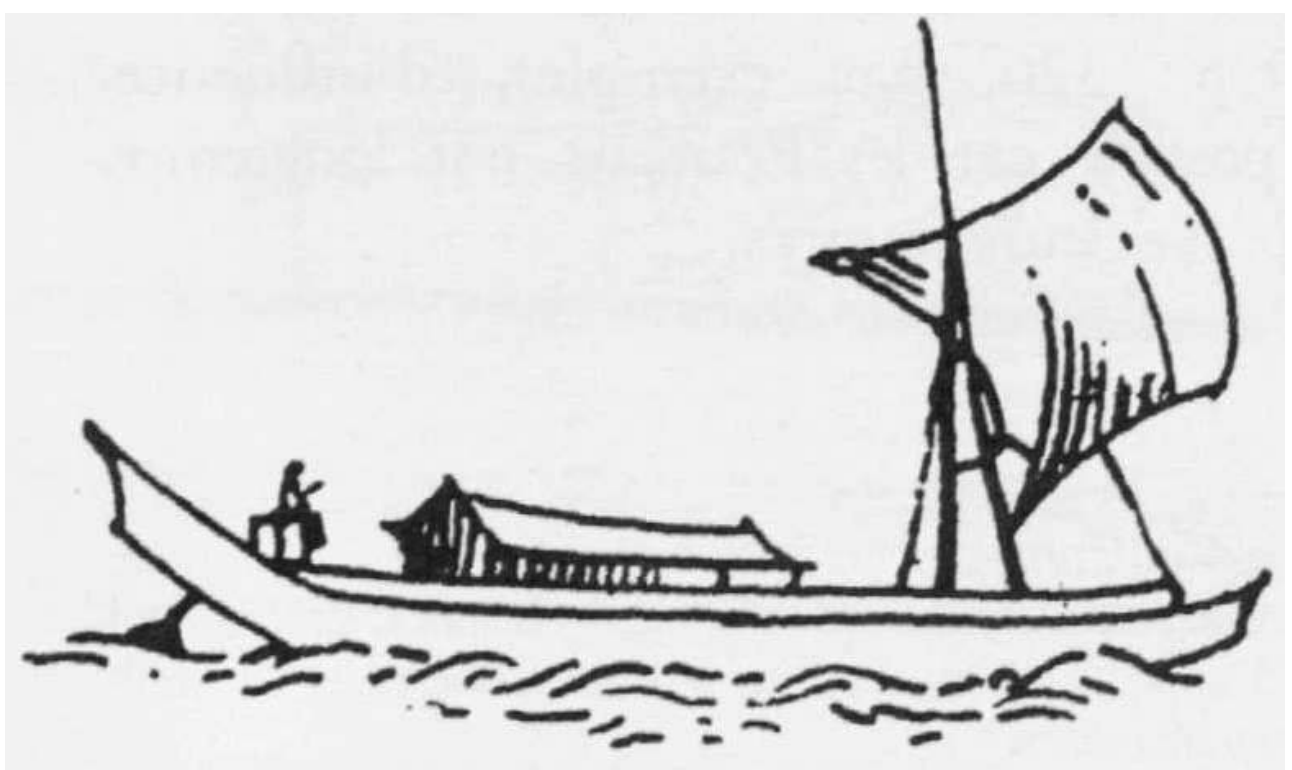

Figure 5. Les pin ko (Myanmar-English Dictionnary 1998 : 277)

Il s'agit d'un bateau construit à clin, qui donne les actuelles formes allongées de ce qu'on appelle ailleurs des long tails. Nous prendrons en considération les bordés pour établir des sous-catégories, qui suivent le développement de la batellerie et des caractéristiques générales de l'évolution d'un bateau, d'abord simple pirogue, enfin utilisée comme transport, puis spécialisée dans le commerce littoral ou hauturier. Ce sont d'ailleurs les bordés qui donnent aux loñ hlè leurs caractéristiques et peut-être même leur nom. Ces bordés sont en saillie (photo 4); ils semblent être des flotteurs intégrés dans la coque. Or, le mot phaung qui désigne les planches des bordés inférieurs et supérieurs peut se traduire (en fonction du ton et des variations régionales que nous ne sommes pas en mesure de donner avec précision) par «flotteur, qui fait saillie »; il peut également s'agir du mot paung qui veut dire "ajouter ». En fait, loñ désigne une pirogue monoxyle, loñ hlè, un bateau avec quille monoxyle et bordés en planches (fig. 6), loñ zat, un bateau fait entièrement de planches. Le pin ko désigne, quant à lui, un bateau de transport à la proue remarquable. Mais le flou demeure entre les termes pin 
et loñ. D'ailleurs les auteurs et les dictionnaires ne sont pas tous d'accord. Ainsi le pin ko devient parfois un pein:go un «dug-out (boat)», selon le Myanmar-English Dictionnary.

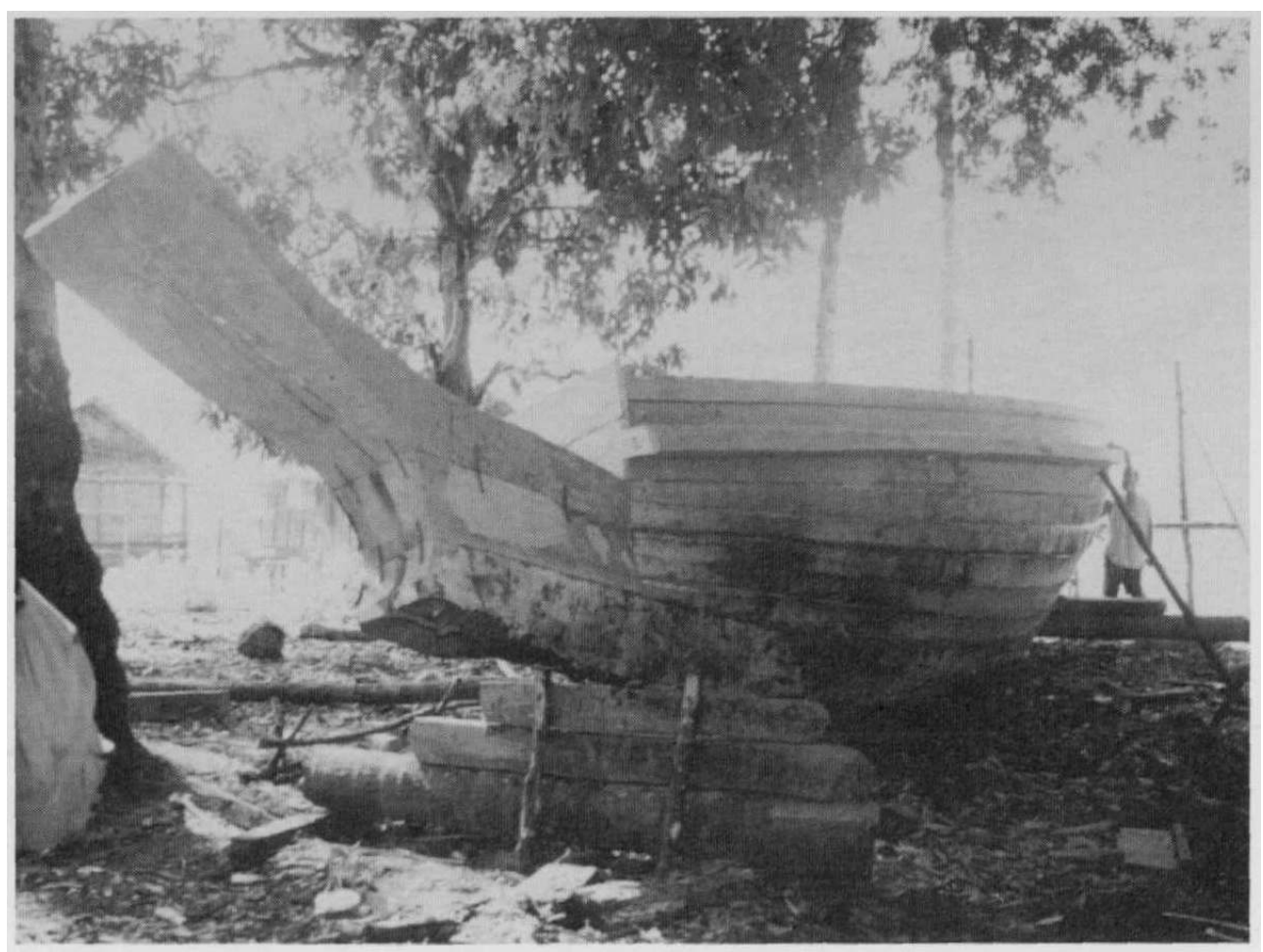

Figure 4. Un loñ hlè en construction sur les îles (cl. MAP, 2000)

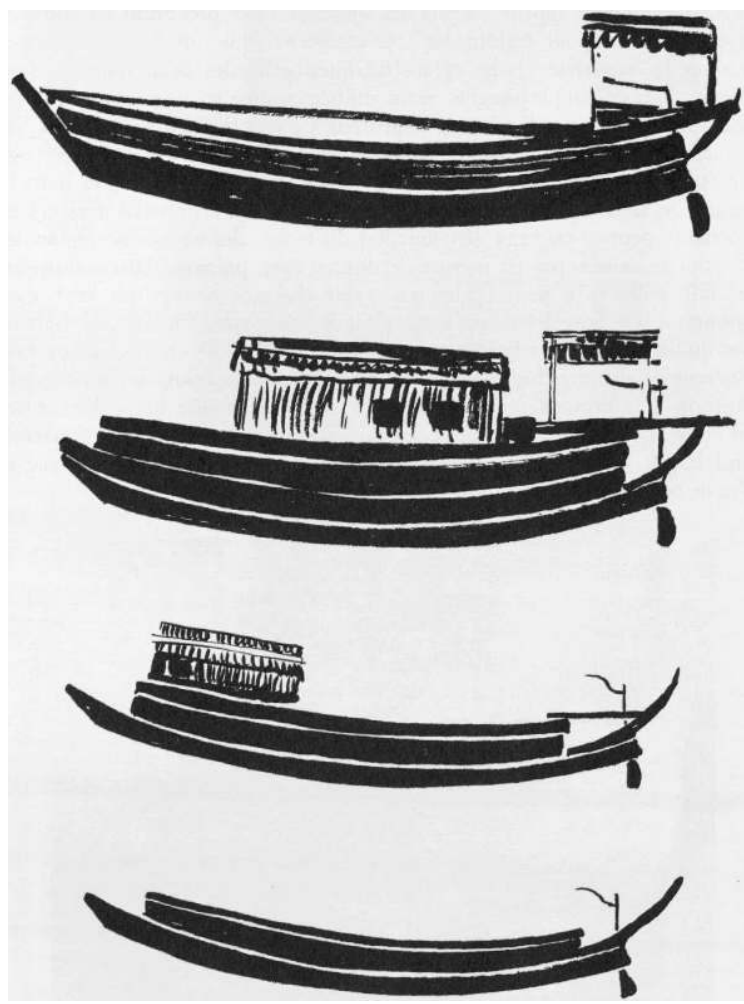

Figure 6. Différentes formes de loñ hlè monoxyles avec des bordés de deux ou trois planches (L. Gansser) 


\section{Structure des loñ hlè}

La proue et la poupe se développent à mesure que le bateau s'allonge et s'agrandit. Certains monoxyles ont donc des proues au sommet évidé en demi-lune (il s'agit des gros bateaux de transports, hnaw par exemple), et toutes sont courbes suite à l'écartement du monoxyle au feu. On doit donc dire que les proues et poupes courbes sont le critère classificatoire d'un loñ, qu'il soit monoxyle ou non. À l'origine, les extrémités s'élèvent à la suite de l'écartement des parois ; par la suite, les charpentiers ont gardé cette forme identitaire. Les loñ sont donc des bateaux longs, à l'origine des monoxyles, qui ont évolué en fonction des besoins de la batellerie birmane. En fait, c'est bien plus la poupe surélevée, dont nous retracerons l'origine et le développement, qui fait leur particularité et au-delà, une référence pour la culture fluviale et marine birmane ; elle a d'autre part des origines symboliques.

Ce monoxyle traditionnel birman est aussi appelé $u$ song pehtut (« en tête, conduire, poupe »), c'est-à-dire ce qu'on appelle localement «le leader des Pehtut», pehtut désignant ici la poupe courbe et élancée, aujourd'hui caractéristique essentielle des monoxyles birmans sur laquelle le barreur, comme le navigateur, avait son poste.

Historiquement, on observera un premier développement du monoxyle avec un pavois simple fait d'un coffrage sommaire de quatre planches; il donnera naissance au loñ ko et permettra le développement du bordé à deux planches chevillées longitudinalement sur les cans et deux autres reliées verticalement à la poupe et à la proue pour fermer ce coffrage par une pièce appelée mokegaton $e^{10}$ que l'on retrouvera sur les loñ hlè. Viendra ensuite le pavois avec varangues sur monoxyles sculptés à la façon birmane.

En règle générale, les bordés des loñ hlè sont constitués de trois planches; la première et la troisième servent à surélever le bordé (fig. 2) et sont logiquement appelées, soit phaung signifiant "être en saillie " ou désignant un encadrement (de porte par exemple, semblable donc à un coffrage), soit paung "ajouter, augmenter " ${ }^{11}$; la deuxième planche, centrale, nommée zat, "joindre ", et par extension «bordé », est utilisée comme virure de maintien, ligne de flottaison ou lisse d'abordage. Elle rappelle le mo des Moken et les anciens flotteurs ${ }^{12}$ des bateaux birmans (fig. 7). Certaines photos (M. et B. Ferrars 1996:160, photo 365) montrent également que les bordés des hnaw ou pin ko peuvent être constitués d'empilements de rondins/flotteurs. Cette pièce de bois ou de bambou, non solidaire du bordé, laisse préfigurer les ceintures de maintien si importantes pour la flottabilité.

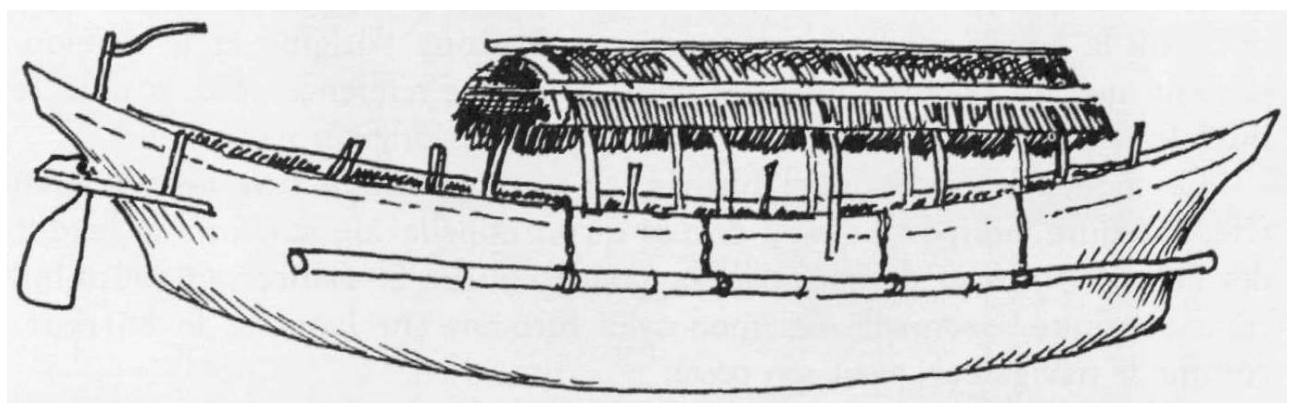

Figure 7. Les flotteurs sont une étape importante dans le développement de la technologie navale ; ici une pirogue de l'île d'Eyles (L. Gansser)

Les flotteurs s'intègrent peu à peu à la coque (le zat des loñ hlè birmans devenant lisse d'abordage, par exemple) ou bien s'éloignent complètement et donnent naissance aux 
pirogues à balanciers. Parfois, pour renforcer la flottabilité du bateau, et pas seulement des pin ko, des flotteurs (bois ou bambou) longent la coque, attachés par des bras de liaison en bois, par des liens de rotins ou de cordes. Nous l'avons observé sur un bateau hybride de kabang moken et de loñ hlè birman de l'île d'Eyles (fig. 7). Les kabang moken et les loñ hlè ont aussi su intégrer cette virure puisqu'elle est devenue la planche centrale des trois bordés (fig. 2).

Certains bordés de ces monoxyles loñ hlè peuvent accueillir, en dessous et au-dessus de la ceinture de maintien "en saillie», plusieurs planches (photo 4). Enfin, d'autres bordés sont formés de trois planches mais ressemblent plus à des bordés à clin « pyramidaux» (fig. 2, photos 2 et 3). Ce bordé renforce la forme effilée du monoxyle et lui permet de fendre les flots avec aisance, l'habitacle étant réduit, mais les cales suffisantes pour la conservation des prises.

\section{Premier inventaire des bateaux d'un archipel oublié}

15 Présentons d'abord le loñ hlè, «bateau monoxyle », le maître birman de l'archipel. Ce loñ hlè est aussi appelé "bateau couché", ce qui indique les choix techniques des Birmans. Ils ont préféré, en effet, réduire la taille de leurs bateaux (d'où le terme " couché » reprenant la traditionnelle classification occidentale en bateaux ronds et longs, J. Poujade 1946 : 186-190), suivant l'évolution économique lente de leur pays. Les constructeurs birmans ont donc su adapter leurs bateaux de pêche au littoral ou à la haute mer, sans faire disparaître le monoxyle.

Lors de nos quatre voyages, nous n'en avons jamais croisé plus d'une centaine en mer, et pourtant nous avons traversé l'archipel sur son étirement maximal nord/sud de $400 \mathrm{~km}$. Certains gros bourgs îliens (sur les îles de Sellore, Kisseraing ou Domel par exemple) ou littoraux (Bokpyin, sans compter bien sur les deux villes de Victoria Point, la ville birmane la plus méridionale de Birmanie, et Mergui qui commande l'archipel) abritent de nombreuses embarcations de ce type, mais partout ailleurs, elles sont dispersées et souvent remplacées par des bateaux de pêche en planches, plus grands.

En effet, parfois les monoxyles loñ hlè cèdent la place à des bateaux de planches construits à l'aide de gabarit, les nga phan hlè ("attraper, poisson, bateau») et à d'autres, aperçus dans les chantiers navals de certaines îles. Ce sont des batu (les bau'tu que nous avons déjà mentionnés), bateaux longs, fins, exclusivement fait en planches, qui rappellent le modèle arabe des dhow, et les effilés et rapides bateaux thaïlandais, long tail, qui font la navette entre le port de la ville de Ranong et celui de sa sœur jumelle birmane, Victoria Point. Entre le long tail et le loñ hlè, les charpentiers de marine ont réalisé un compromis: une embarcation plus longue, plus élevée, plus large à l'avant qu'à l'arrière (on sent ici l'influence de l'occident et du monde arabe), mais qui garde toujours la poupe surélevée, spécificité birmane (influence du pin ko). Ils sont destinés à la pêche au filet et construits dans les petits chantiers navals présents dans toutes les bourgades de quelque importance. Il est utilisé d'abord par les Karens, très nombreux à naviguer dans les eaux de l'archipel et à vivre sur les îles puis par les Birmans, plus présents sur le littoral et les grosses îles proches de celui-ci. Ce bateau peut être long de 15 à 30 mètres. Aucune particularité ne peut lui être attribuée, sinon la simplicité de sa construction et son efficacité dans les eaux calmes de l'archipel à la saison sèche. Ce bateau semble être une extension du loñ hle (fig. 8). 
bateau à voile et à coque bordée opposé aux pirogues monoxyles des Birmans. Ce
sanpho, d'origine malaise, est paradoxalement le seul à être mentionné dans les
inscriptions (Luce $1960: 336$ et 362). Il a disparu des îles, subsistant dans quelques
bourgs littoraux (Bokpyin par exemple), et adoptant, autant que nos enquêtes partielles
nous autorisent à le dire, les modèles de bateaux birmans ${ }^{13}$.

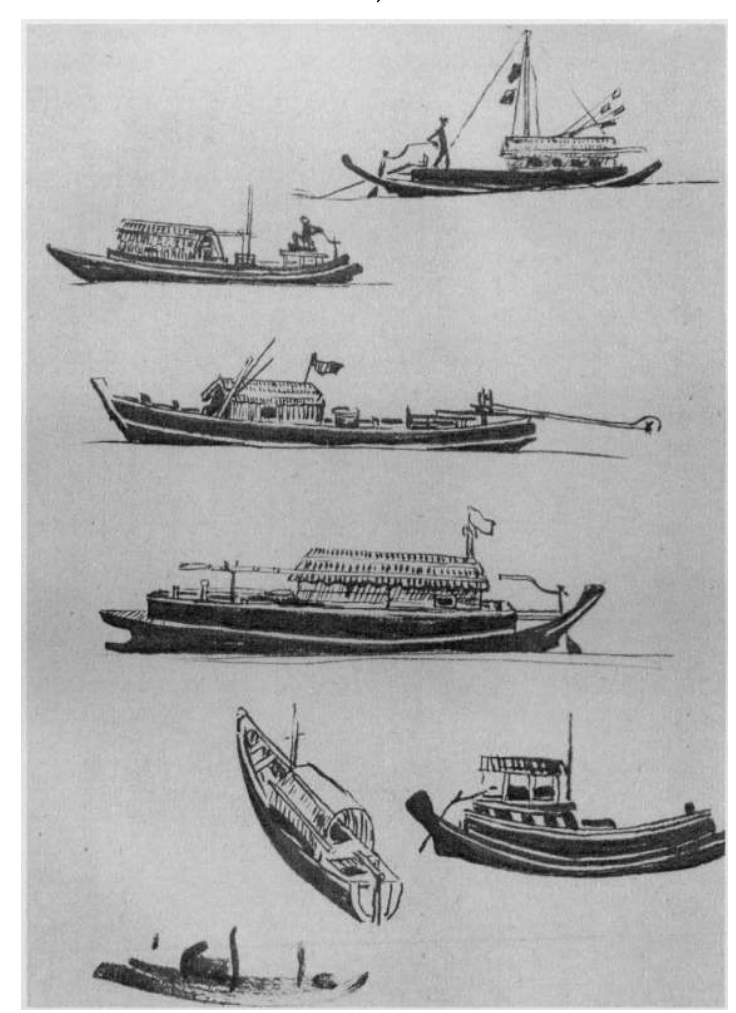

Figure 8. Deux sortes de loñ hlè, un bateau inspiré d'un long tail et adapté à la pêche, un bateau moken avec une poupe birmane, un nga phan hlè et un autre loñ hlè L. Gansser)

Techniques \& Culture, $35-36$ | 2001 


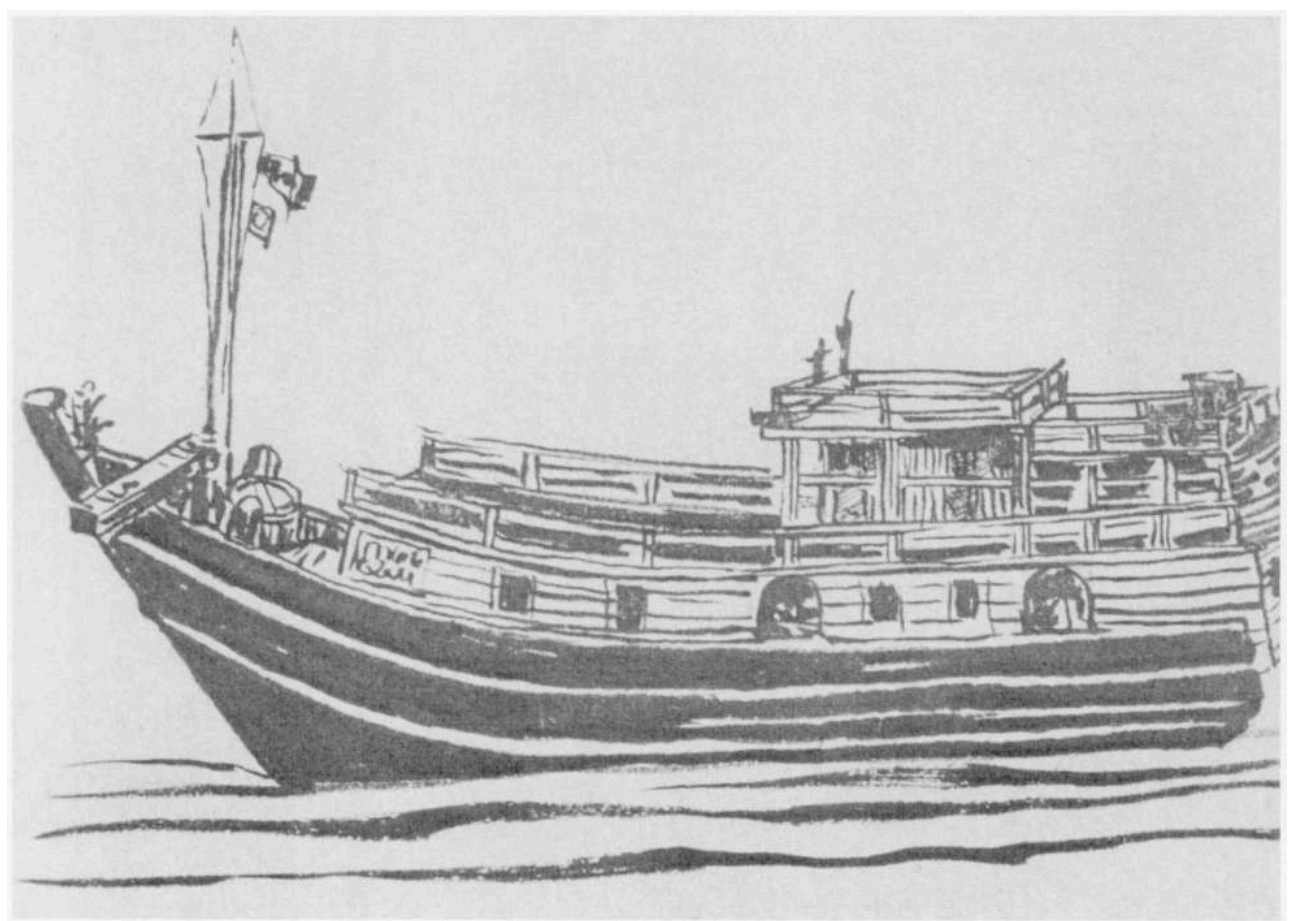

Figure 9. Un cargo birman (L. Gansser)

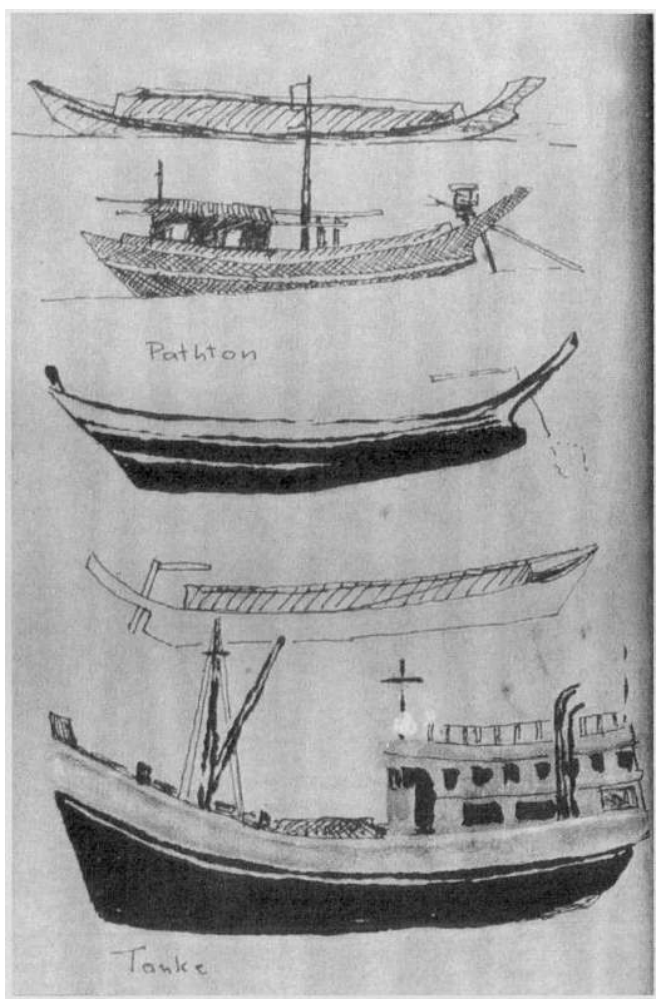

Figure 10. Différentes variétés de bateaux croisés dans les eaux de l'archipel Mergui (L. Gansser) 


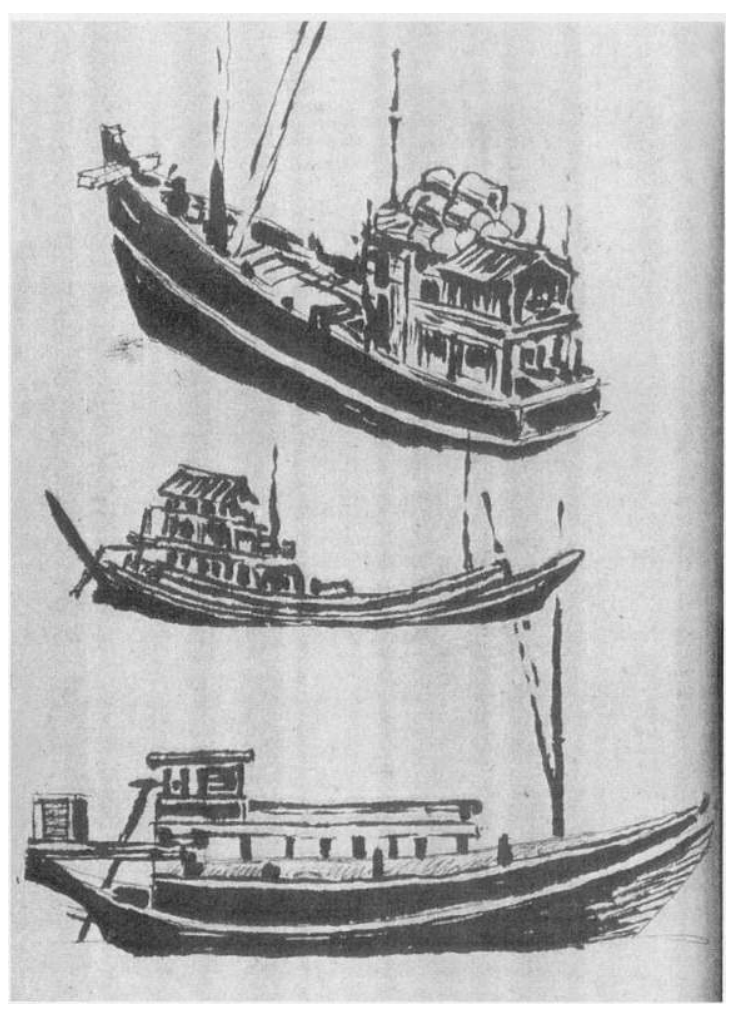

Figure 11. Les gros bateaux de pêche modernes, d'influence thaillandaise (L. Gansser)

Les praos malais croisés par T. Forrest (1792: 5 et 12) au XVIII ${ }^{e}$ siècle ont longtemps été les maitres des îles. Mais ces bateaux rapides, razziant les esclaves et tout autre bien échangeable sur les marchés de Singapour et Pinang (Ivanoff 1998), se sont vite transformés en bateaux de commerce et de transport. F. Robinne les considère comme tels et les seuls exemples que nous avons vus sur l'île de Singaye (île située juste en deçà de la frontière birmano-thaïe), étaient de gros bateaux de transport, de plusieurs dizaines de mètres de long et très évasés, à fond plat. Ils ont aujourd'hui complètement disparus, remplacés en Thaïlande par les long tails et en Birmanie par les loñ hlè.

Les jonques de facture birmane ${ }^{14}$, utilisées pour le transport, demeurent les témoins d'un monde oublié et surtout prouvent la vivacité de l'influence de la Chine, déterminante sur la construction navale birmane. Leurs voiles sont lattées, leurs poupes, larges et hautes, et leur gouvernail, latéral, caractéristiques de la construction asiatique prenant son modèle sur les oiseaux. Les références aux oiseaux à propos des embarcations expliquent, par exemple, les commentaires sur «l'envolée du perroquet ", ou la forme de canard utilisée pour décrire d'abord les sampan à double poupe (photo 1) et ensuite tous les autres bateaux. Ce choix asiatique offre les lignes essentielles des bateaux birmans, avec un arrière plus lourd et plus large que l'avant, à l'image des corps de canards (Poujade $1946: 210-211$ ). Cette influence chinoise existe un peu partout en Asie du Sud-Est, en Thaïlande par exemple :

" The reua pet has a smooth, rounded bilge so it can skim over mudflats. It also has a square transom. The bow has painted eyes in the traditional chinese manner, and the longtail engine is mounted amidships on the gunwale. The boat is steered by a paddle lashed to the transom. The eyes are looking downward, on these duckboats, to bring the fishermen good luck with a big catch, whereas on the Chinese trading junks the eyes look forward towards the port of destination. » (D. Slee, n.d., p. 24) 
Décrivons alors succinctement ensuite les sampan ${ }^{15}$ (fig. 12), sortes de jonques chinoises omniprésentes en Asie du Sud-Est et adaptées par les populations qui les ont acceptées. Ce mot serait, pour certains informateurs birmans, la transcription chinoise de "trois choses »: 1) coque, 2) bordé bâbord, 3) bordé tribord. Nous distinguerons deux sortes de sampan birmans qui rappellent les «pirogues en cinq parties » dont parle J. Poujade (1946: 186).

A) thanvan hlè : petit bateau dont la double poupe représenterait un perroquet en train de s'envoler (fig. 12, premier dessin). Il s'agit du modèle courant de petit sampan, que l'on retrouve de la Chine du Sud jusqu'à Yangon en passant par l'Indonésie. Ce modèle, manoeuvré à la godille et parfois à l'aviron, est utilisé pour le transport de passagers d'une rive à l'autre d'une rivière (calme) ou d'un bateau à un quai. Il sert également à charger et à décharger les marchandises. Ces bateaux de transbordement, de faible tirant d'eau, sont longs de 5 ou 6 mètres, à fond plat et en planches. Ils étaient autrefois utilisés comme bateaux de pêche (en tout cas en Thaïlande du Sud il y a encore trente ans) et même comme bateau de guerre (Charney 1997 : 42). Les extrémités de la double poupe courbe du petit sampan se rejoignent parfois, formant une arche. Cette double poupe, quand elle ne se rejoint pas, a offert un modèle pour les barques royale à double poupe (quand celles-ci ne sont pas faites de deux monoxyles).

B) thavangyi : gros bateau à double poupe (fig. 12, dessins 2 à 6). Il s'agit d'un modèle, copie de jonque chinoise, qui peut atteindre 40 mètres de long; un gros bateau de transport donc, avec une cale gigantesque et un château arrière relevé dominant le chargement.

On mentionnera aussi le kattu, en planches, avec gouvernail axial, propulsion à rame et à proue convexe, avec lequel des équipages de cinq à six hommes faisaient régulièrement voile pendant la mousson du nord-est jusqu'aux îles Nicobar (Ferrars $1901: 138)$. 


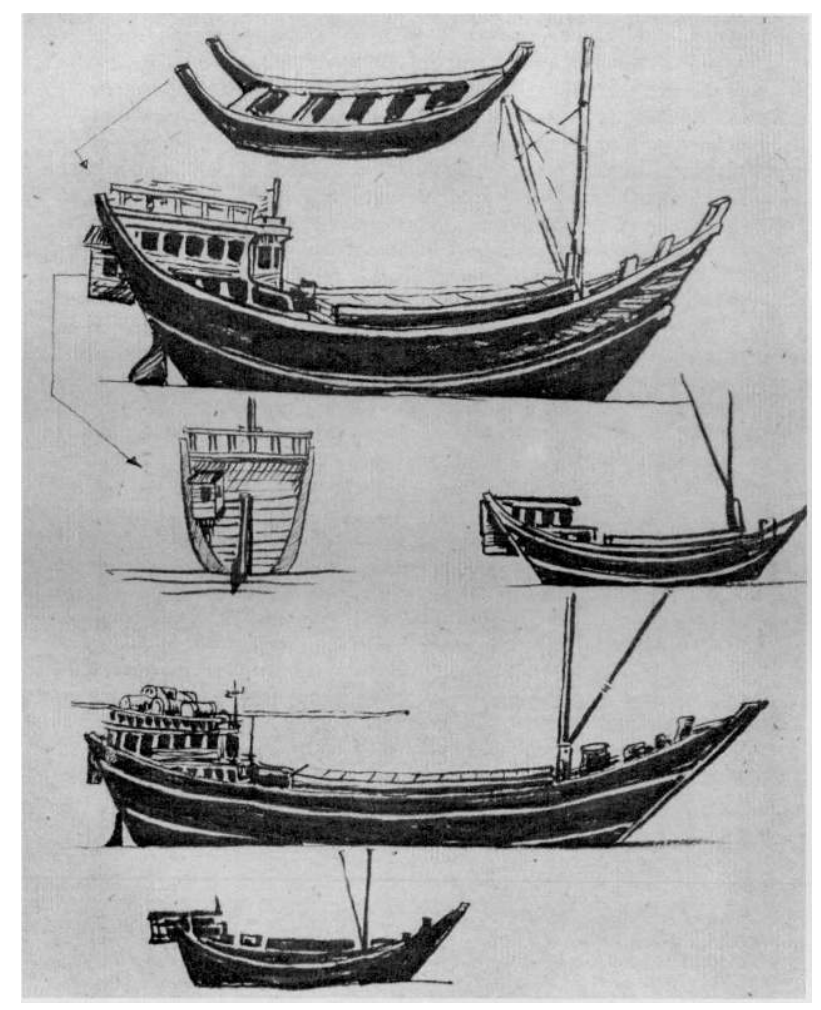

Figure 12. Les jonques et sampan birmans (L. Gansser)

\section{Le monoxyle loñ hlè}

C'est un rare privilège que de pouvoir caresser les coques monoxyles des loñ hlè, apprécier leur solidité, leur fermeté et leur douceur tout en parcourant de l'œil les tontures élégantes et les couleurs sombres de ces seigneurs issus des sylves environnantes (Hopea, Shorea, teck...). C'est une expérience agréable et presque sensuelle, car peu d'endroits au monde offrent encore aux amateurs de bateaux la possibilité de voir des énormes fûts de 10 à 30 mètres abattus puis métamorphosés en pirogues $^{16}$. La profusion des bois, la variété des essences locales, le petit nombre d'habitants perdus au milieu de ces 800 îles font que le monoxyle est encore le roi ici. Plutôt que d'élever les bordés, ou construire des coques en planches par exemple, les pêcheurs birmans ont choisi de conserver le monoxyle alors même que son ancêtre pin ko s'allongeait et se rehaussait. La Birmanie n'a pas voulu développer ses capacités de production et d'exportation au maximum de ses possibilités et les résultats dans le domaine de la technique navale s'en ressentent. La Birmanie a choisi l'équilibre et non le développement incontrôlable de la production (et donc la construction de gros bateaux de pêche, pourtant présents, $c f$. fig. 10 dessin 5 et fig. 11 dessin 1) qui aurait empêché la fabrication des loñ hlè, moins longs, moins hauts mais mieux adaptés à la pêche en mer que leurs ancêtres loñ ko et pin ko. De plus, les difficultés d'approvisionnement et l'absence de matériaux de base imposent un travail avec des outils limités et multifonctionnels comme l'herminette et la hache, voire la doloire.

Nous n'avons pu observer directement les premières phases de construction du monoxyle (abattage, évidage, écartement des parois). Mais l'abattage, si l'on en croit les informateurs, se fait d'une manière classique, à la scie électrique et autrefois à la hache, 
les bûcherons montant sur des plates-formes en bambou ou en bois pour éviter de couper les empattements inutiles dans la construction (pourtant utilisables pour la réalisation des poupes). Comme partout en Asie du Sud-Est, les arbres abritent des esprits qu'il faut se concilier.

«Les arbres ont dans l'imaginaire birman (et au-delà) une grande importance. Parmi les naq de la nature non-personnifiés qui restent une catégorie actuelle du monde spirituel, ceux des arbres sont particulièrement respectés : ils sont connus sous le nom d'origine pâlie de yôkke'sô. D'autre part, les dewatau sotâpan môns dont le culte existait parallèlement à celui des naq, étaient des divinités demeurant dans les arbres (Shorto 1967: 128). Ce contexte dans lequel les arbres, pourvu qu'ils soient beaux et vieux, focalisent de nombreuses pratiques cultuelles, éclaire la convergence entre l'accent mis sur le champak dans l'histoire du forgeron et l'explication répandue en Birmanie selon laquelle le cuire du naq domestique serait une substitution du culte autrefois rendu aux arbres abartus pour construire une maison. » (Brac de La Perrière 1989 : 24).

On retrouve ce culte sur les bateaux et on dira simplement ici que le culte de la maison et du nat domestique a été transféré sur le bateau. Le monoxyle est légèrement concave, ce qui est dû à l'écartement de ses parois (à l'aide de barrotins d'écartement, daukkwa), après son passage au feu destiné à l'assouplir. Pour plus d'informations sur l'évidage et les opérations techniques suivantes concernant le monoxyle, on se reportera par exemple à la description de M. et B. Ferrars (1901 : 133-134).

Nous ne décrirons que très succinctement le gréement ${ }^{17}$. Tous les loñ hlè ont une voile supplétive, en toile de jute, en nylon, pour pallier les dysfonctionnements du moteur. Il s'agit d'une voile à bourcet, à l'image de la voile moken mais qui était autrefois réalisée en tissu (Holbrook et Manoh Suriya 2000 : 262).

Le loñ hlè n'est pas échancré, fait remarquable qui le différencie des autres embarcations. Sa poupe surélevée le relie pourtant à ses ancêtres pin ko et loñ ko. Les yeux peints ou sculptés que l'on trouvait sur toutes les proues des monoxyles ont disparu; ils décorent et protègent essentiellement les bateaux en planche et les jonques, cela tendant à prouver l'apparition récente des loñ hlè. Pour F. Robinne (1995: 59) :

«La proue seule fait exception puisqu'elle est linguistiquement assimilée à une tête

- humaine ou animale - dont la représentation minimale est l'oeil qui est parfois symbolisé sur chacun des flancs : œil d'oiseau ou de poisson parfois, oeil de naga, de galon ou de belu plus souvent, yeux terrifiants toujours, représentés aujourd'hui encore sur les pirogues de course comme sur certains bateaux de commerce, et destinés, dit-on, à épouvanter l'embarcation adverse au point de la faire chavirer ; yeux mythiques qui mettent la traversée des océans sous la protection de divinités qui habitent le monde sous-marin et qu'il convient de se rendre propices. »

Les pièces $\mathrm{du}$ bateau birman ne sont pas linguistiquement issues d'un modèle anthropomorphe, fait remarquable en Asie du Sud-Est. Sur la "tête » émergée du monoxyle, en avant de la fermeture du bordé à trois planches, se trouvent les offrandes «temporaires » (disposées avant chaque départ puis retirées bien que des fleurs ornent souvent cette étrave au mouillage) et le siège de la "vigie ». Il s'agit de la partie pleine du monoxyle, qui renforce sa puissance de pénétration dans l'eau. Le bordé se ferme à l'avant et à l'arrière par une planche horizontale, installée là où le monoxyle commence à être évidé. Cet ancien pavois (développement des fargues utilisées sur les pirogues monoxyles fluviales), dont nous parlerons plus loin, est transformé en bordé en planches et ressemble à un coffrage (fig. 2 et fig. 13). 

du monoxyle avec pavois utilisé sur les fleuves. Sur ces pirogues aux échancrures déjà marquées (mais qui disparaîtront par la suite), un homme est assis en avant du pavois, chargé de diriger le bateau et d'éviter les multiples incidents qui peuvent survenir à la surface.

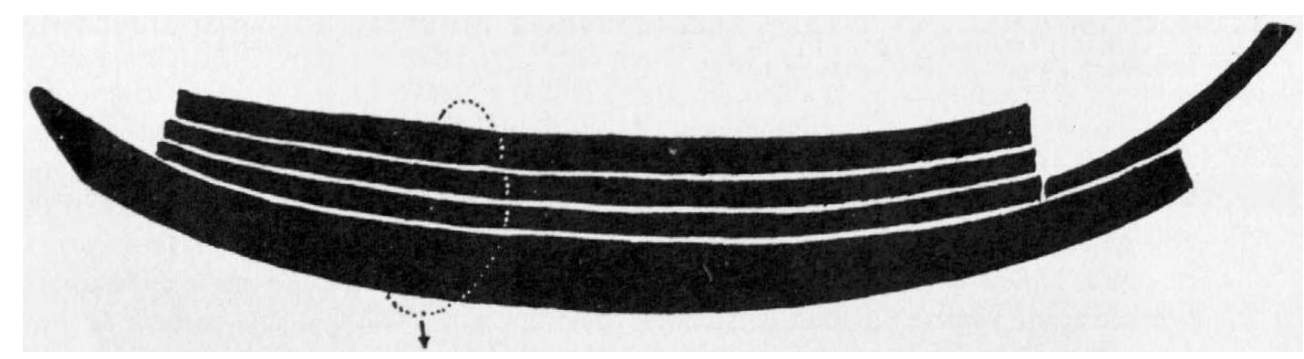

Figure 13. Le loñ hlè ; bordé en forme de coffrage (L. Gansser)

L'éperon inférieur de l'échancrure que domine ce marin est coupée pour aider à la stabilité du bateau et éviter -ou amortir- un choc frontal susceptible de le faire déjauger. La perche de propulsion est maniée à partir de l'éperon supérieur de cette échancrure. Cette fonction de vigie a été conservée sur les loñ hlè. Pour elle, deux baguettes de $50 \mathrm{~cm}$ environ de bois sont chevillées transversalement sur la proue. Elles servent à protéger le marin en charge de la surveillance de la mer, guettant de ce poste avancé les bancs de poissons et l'état de la mer ; il est ainsi mieux « arrimé ». Cette vigie doit également frapper la mer avec des longs bambous et des morceaux de bois pour rabattre les poissons vers les filets.

Les échancrures birmanes, sans être aussi prononcées que celles des bateaux moken et n'ayant, semble-t-il, pas autant de valeur symbolique, n'en demeurent pas moins très marquées bien que l'éperon inférieur soit coupé. Les loñ hlè n'ont pas d'échancrures, ni à la proue, ni à la poupe. Leur poupe est cependant surélevée par une pièce de bois courbe qui vient l'épouser dans sa partie supérieure (photo 4) et qui forme un éperon pour accueillir le gouvernail axial, ce qui les rattache aux pin ko.

-être que cette absence d'échancrure, à la base de la poupe en tout cas (c'est-à-dire l'extrémité du bloc monoxyle), permet de la renforcer en laissant au monoxyle un rectangle de bois plein non affiné d'environ $20 \mathrm{~cm}$ sur 20 (fig. 14, photos 5 et 6). Il s'agit de consolider l'endroit où frappe le gouvernail agité par les courants, les vagues ou la houle.

La poupe confère au loñ hlè son aspect tout à fait particulier qui le différencie des autres bateaux et permet de retracer sa lignée. Elle exprime la spécificité du bateau birman en général, car elle fixe l'art et les conceptions esthétiques des marins. Le creux de cette envolée, sculpté de motifs variés à son extrémité la plus haute, longue engoujure étroite de plusieurs dizaines de $\mathrm{cm}$ sur quelques $\mathrm{cm}$ de large (dimensions variant en fonction de la taille de l'embarcation et donc de son gouvernail) reçoit l'engoujure du gouvernail rétractable (qui permet de le retirer en présence de bancs de sable et qui peut aussi amortir les choc des hauts fonds) et axial (et non latéral, disposition qui relierait le bateau birman à l'Inde, Robinne 1995).

Sur ce bras courbe et élancé, le barreur peut se tenir debout et regarder ainsi pardessus le château arrière bien que le siège du pin ko, jadis richement décoré (photo 7) ait été remplacé par une simple planche transversale et que les décorations soient descendues sur la poupe (fig. 15). 


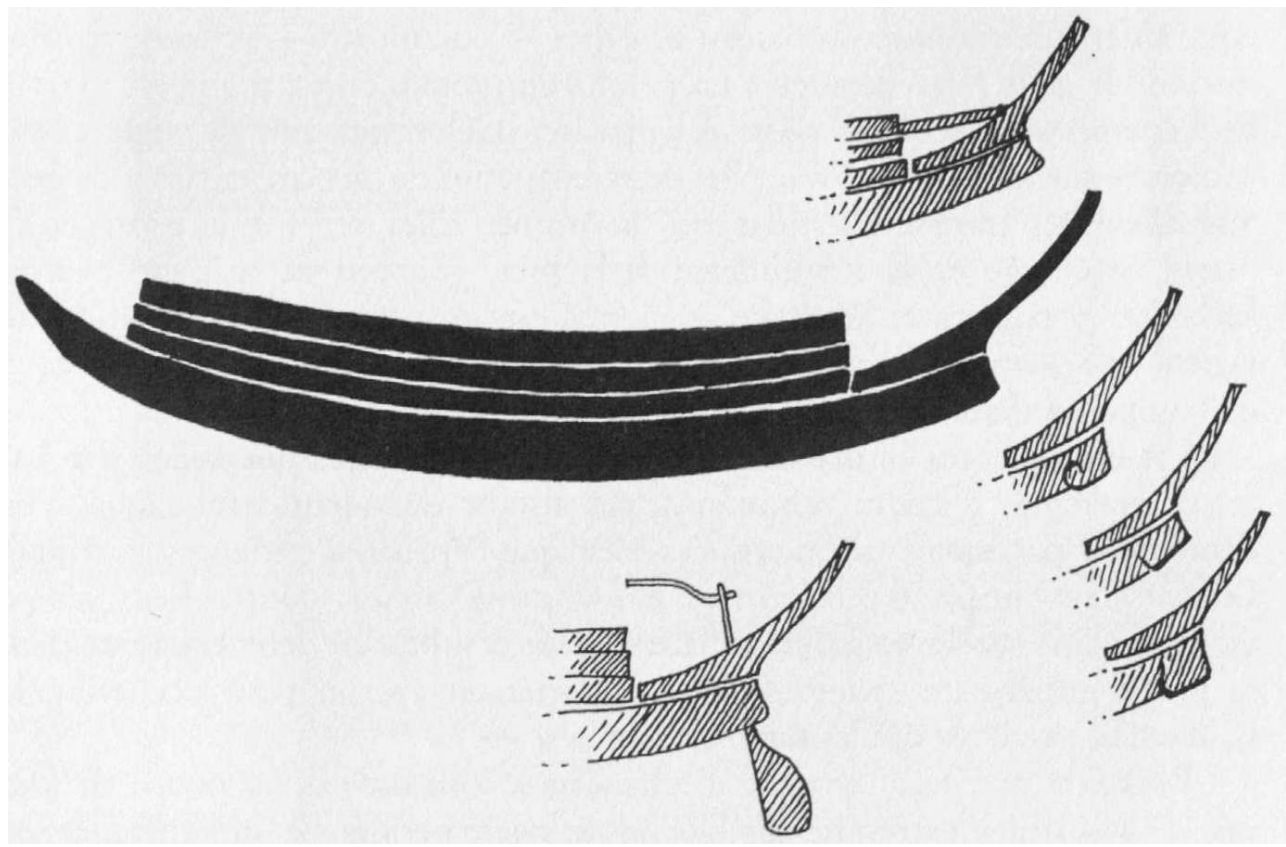

Figure 14. Structure du loñ hlè monoxyle, trois planches formant bordé, poupe courbe, renfort de bois plein à l'arrière du monoxyle (L. Gansser)

Ces décorations sont en accord avec le nouvel environnement conquis par les marins ; on y découvre ainsi des figures marines (crevettes par exemple) mais aussi et encore des motifs floraux rappelant les $k a n o u^{18}$ des temples et des sièges des pin ko (photo 8). Cette prolongation de la proue, qui peut s'étendre sur deux mètres, serait, selon les pêcheurs interrogés, due à la copie des modèles de bateaux de course. Cette référence est intéressante, les bateaux de course étant des objets rituels d'eau douce richement décorés. Ce lien avec les pirogues fluviales et rituelles permet de retracer certaines constantes dans les domaines des techniques et des croyances, notamment celles concernant la poupe, surélevée pour permettre, à l'image des jonques, de surveiller la mer par-dessus la cargaison (qui nous rappelle le barreur des pirogues anciennes). 

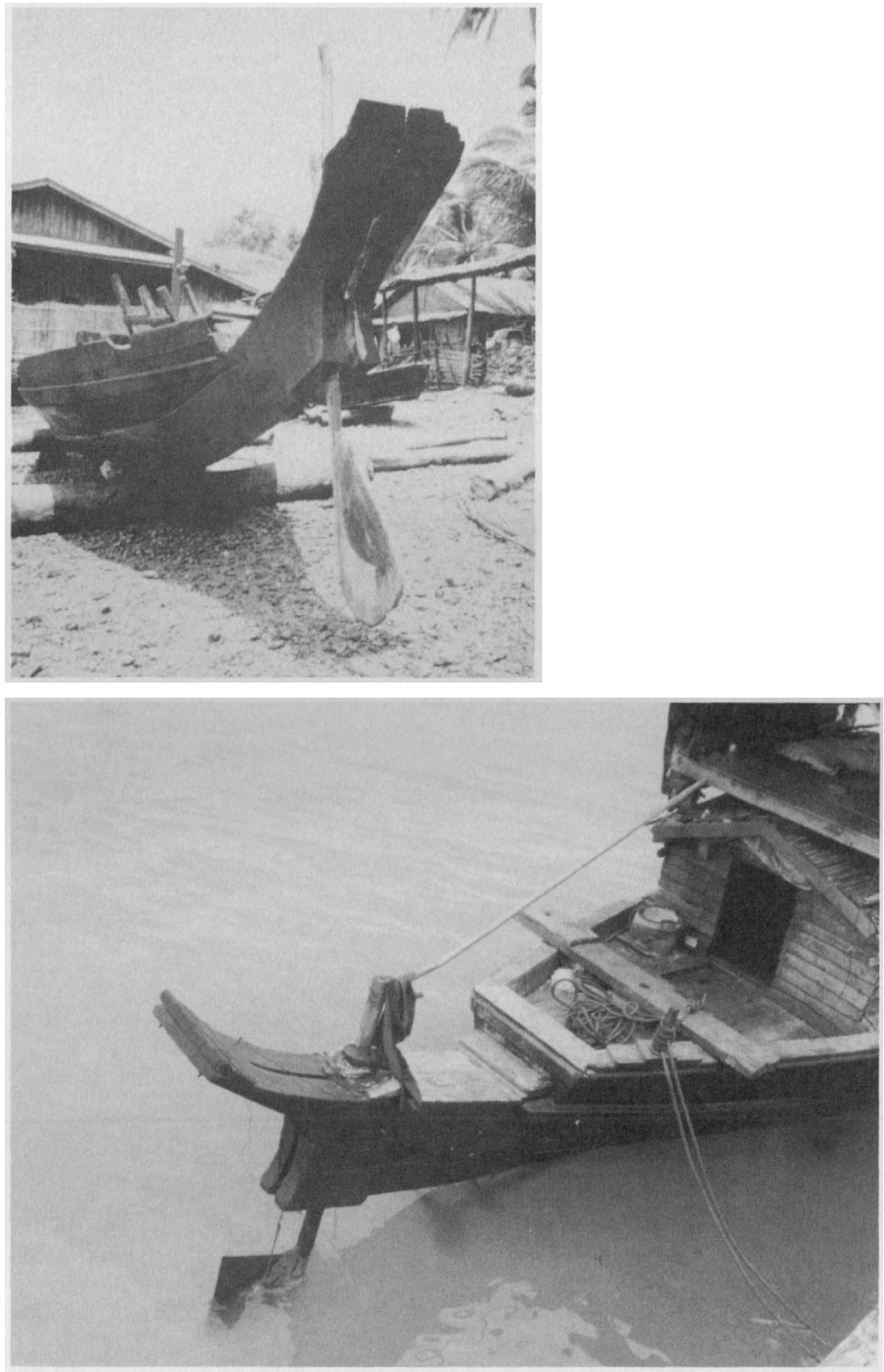

Photos 5 et 6 . Poupes avec gouvernail axial rétractable et hélice centrale perçant le monoxyle (cl. MAP 1997) 


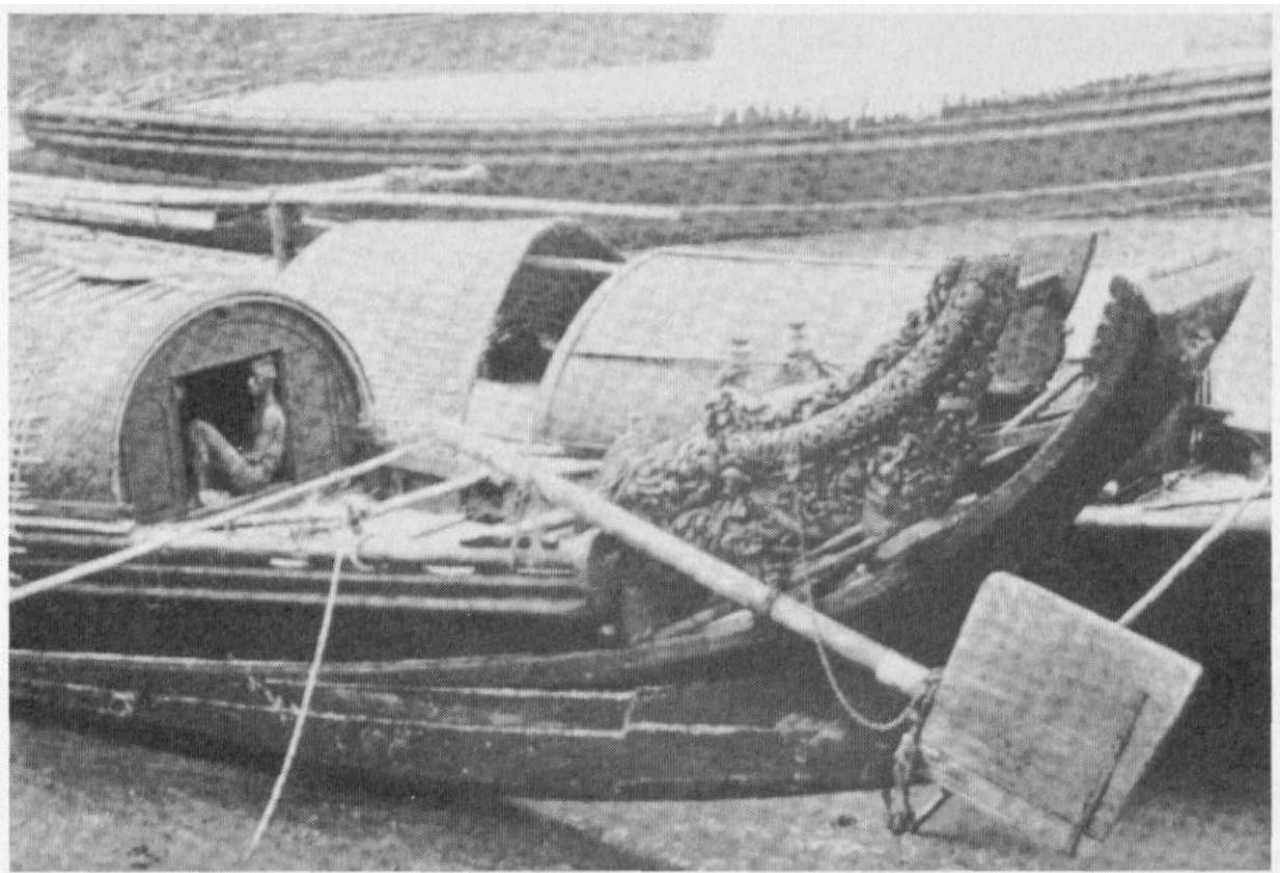

Photo 7. Les décorations sculptées en bouquet floral des sièges des barreurs. Autour du monde. Aquarelles. Souvenirs. Voyages (cl. XII)

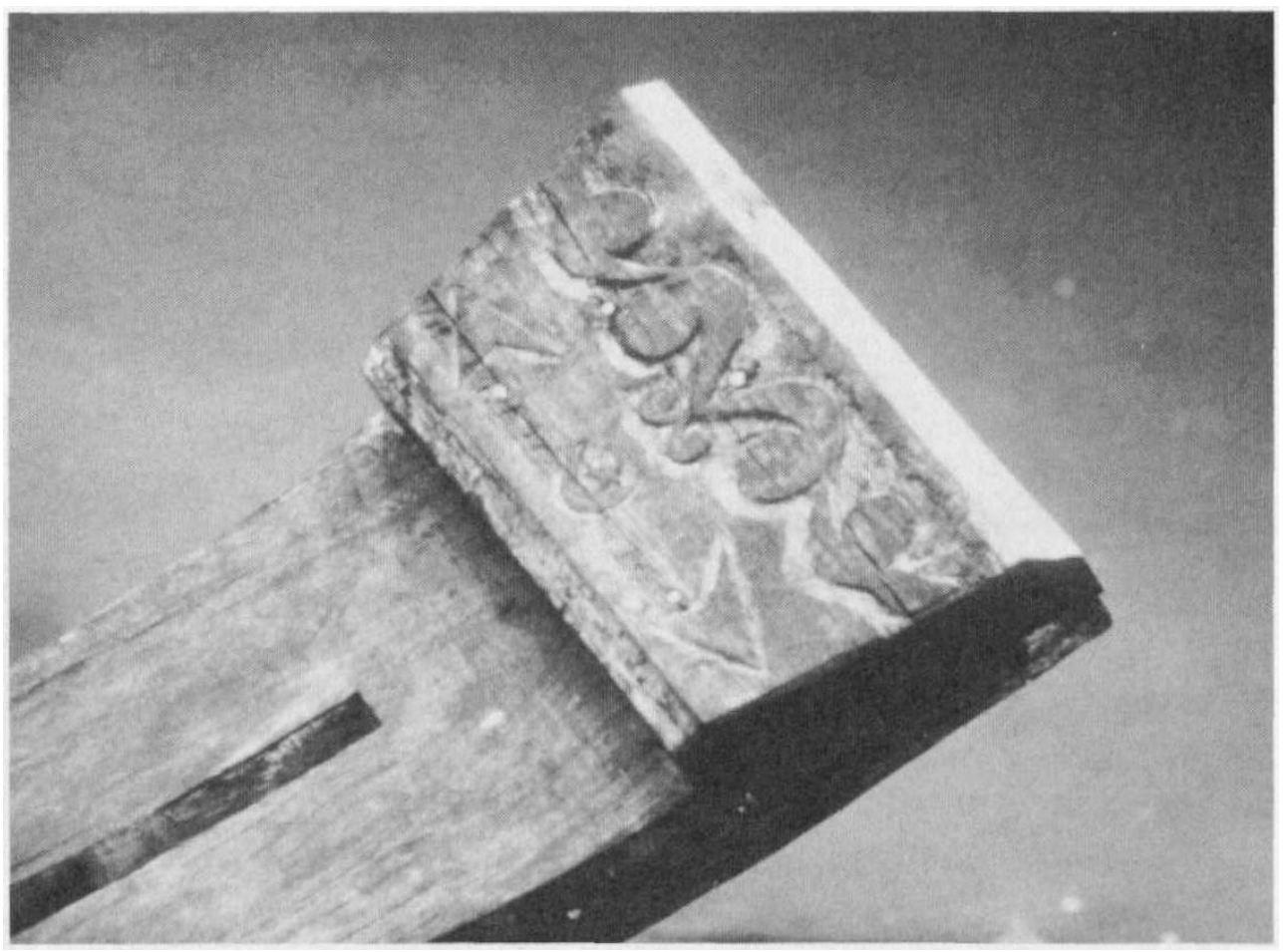

Photo 8. Les décorations de la poupe des loñ hlé 


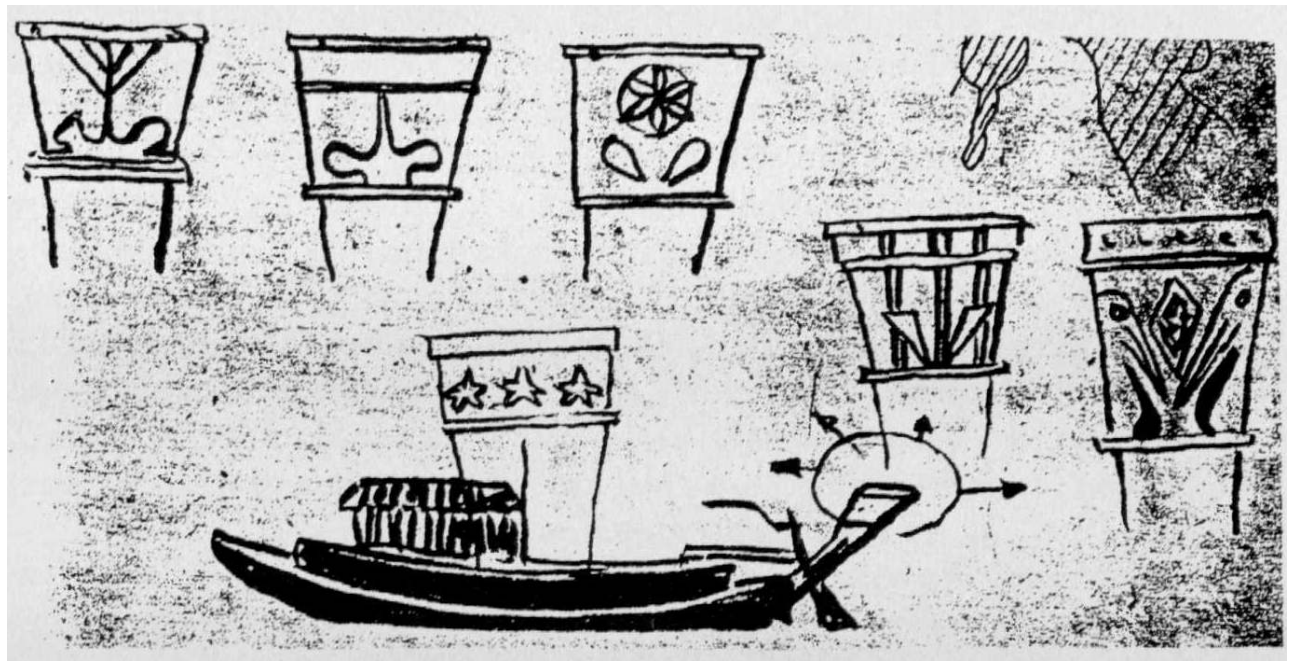

Figure 15. Décorations florales et animales sur les poupes (L. Gansser)

Certains loñ ko peuvent être dirigés avec une godille faite d'un aviron tenu par le pied et la cheville, ce qui n'est pas sans rappeler le maniement d'un aviron/gouvernail par des marins du lac Inle ${ }^{19}$. Le bateau monoxyle de ce fameux lac est parfois surmonté d'un petit pavois de protection, ou parfois de simples fargues; ce pavois est aussi fin et bas que ceux d'une pirogue de course. Cette première élévation est une méthode d'amélioration classique des qualités nautiques des pirogues. Ce pavois est souvent, et d'abord, constitué de quatre planches qui font le tour du tronc creusé comme les coffrages des futurs loñ hlè. Sur ces pirogues, la partie supérieure de la proue et de la poupe prolonge la coque mais fait encore partie du bloc monoxyle. Effilée dans sa partie inférieure, elle donnera naissance à la future échancrure birmane. L'évidage, à la proue et à la poupe, est progressif, en pente douce jusqu'au fond du monoxyle. Cela nous rapproche des premières pirogues indonésiennes (des îles Célèbes, sur le lac Tondano) et des pirogues en " $\mathrm{U}$ » de Bornéo, dont les extrémités sont fermées par une planche semi-circulaire (Neyret $1974: 189$ ).

On retrouve cette planche fermante en demi-lune (qui rappelle la proue des hnaw birmans) à travers tous les pays de l'Asie du Sud-Est. Il s'agit de séparer le monoxyle évidé de la proue et de la poupe et de protéger les marchandises tout en offrant sur la partie élevée un siège de vigie pour un homme. Cette embarcation donne naissance à la pirogue fluviale classique, utilisée pour les déplacements, les collectes et les ventes « à domicile ». Une autre forme existe parallèlement : la pirogue monoxyle évidée en pente douce à la proue et à la poupe. C'est ce dernier modèle qu'ont choisi les Birmans. Cette pente intérieure assure une meilleure stabilité de l'embarcation, des lignes plus fines, et autorise le développement des futures poupes élancées. Elle permet aux hommes (barreur, pécheur, pagayeur, marchand, etc.), de s'y installer, accroupis ou debout.

Bien souvent, des «fausses membrures", renforçant la coque monoxyle et permettant l'installation de sièges ou bancs de nage, sont installées. Elles permettront de mettre les allonges et d'élever les bordés par la suite. 


\section{L'influence des barques royales}

sur la construction navale populaire est évidente ; reportons-nous à la description de $\mathrm{H}$. Yule $(1860: 259)$ :

«La députation était escortée de cinq ou six canots de guerre : c'était la première fois que nous rencontrions ces immenses embarcations; l'avant en est très bas et très fin, l'arrière très élevé se recourbe au-dessus de l'eau ; les rameurs, au nombre de quarante à soixante, sont deux sur chaque banc: tout l'extérieur du canot est doré, et toutes les rames le sont aussi. Les matelots, vigoureux et robustes, portaient tous leur conique chapeau de bambou. [...] Des bandes de mousseline et des filets couverts de clinquant ornent les poupes élevées des canots de guerre, où flotte avec grâce une grande bannière blanche bordée d'argent, sur laquelle s'étale le blason de l'empire, un paon grossièrement dessiné. Souvent à côté de l'oiseau oriental, une carafe européenne sert de pomme de mât de bambou auquel s'attache le pavillon. C'est un ornement très en faveur chez les Birmans, et parfois même une modeste bouteille de Seltz, domine la pointe extrême des pagodes. Un court mâtereau dressé à l'extrémité de la poupe des canots de guerre porte le $h t e e^{21}$, cet emblème royal et sacré. Ce n'est pas à l'arrière, comme en Europe, mais à l'avant du canot, sur une espèce de petite plate-forme, que se place le personnage le plus important du bord."

C'est plus qu'une parenté cependant, car les types populaires et royaux de construction puisent leur source ultime dans les constructions de pirogues rituelles; et leur association avec les dragons aquatiques est évidente. Nous ne nous attarderons pas sur la description des proues royales, mais nous ne pouvons pas les occulter car leur puissance symbolique a entrâné les croyances populaires et dessiné les lignes 
essentielles des bateaux de pêche et de transport. Ces barques royales, aux noms rappelant les légendes de légitimation de la royauté le plus souvent, donne au roi une ascendance divine. Le bateau devient le véhicule des rois, montures des dieux, mises à la disposition des souverains. La symbolique est donc forte mais n'est pas aussi puissante dans la construction navale populaire. Ces embarcations rappelant les grands thèmes de la mythologie bouddhiste et indienne se retrouvent aussi bien en Thaïlande qu'en Birmanie. Elles représentent par exemple un naga (un dragon mi-aquatique, miterrestre, voire céleste) ou un makara, créature fantastique, hybride entre crocodile, éléphant, poisson, etc., monture de Varuna, ancien dieu du ciel devenu maître de l'océan. Les défenses de makara sont parfois interprétées comme des éperons destinés à frapper les bateaux ennemis. (Pour une discussion sur l'utilisation pratique et technique de cette représentation religieuse et symbolique Charney $1997: 43$ ).

Cette proue prend la forme d'un éperon, concave ou convexe selon l'utilisation des bateaux. Elle permet à certains auteurs de classer les bateaux bien que, nous l'avons vu, leur poupe soit d'une plus grande pertinence pour l'établissement d'un inventaire et que la forme reste le déterminant principal. Ces proues, certes moins décorées et élégantes que les poupes, ont aussi une portée symbolique et esthétique (figs. 16 et 17), comme ailleurs dans le monde océanien et austronésien (Neyret 1974; Coiffier, ce volume).

Les courses de bateaux ont une origine si lointaine et si commune à tous les peuples de l'Asie du Sud-Est qu'il est difficile de retracer leurs origines mais l'idée essentielle est de mettre en scène le conflit des saisons et le pouvoir des rois sur les éléments naturels. Les rois birmans se sont considérés comme les descendants de la course du soleil (Adicca Vamsa) et à ce titre, ils présidaient les cérémonies du premier sillon et les courses de pirogues (rite agraire et de fertilité symbolisant le changement des saisons).

Dans la littérature et les chroniques, 36 sortes de bateaux de cérémonie, 30 sortes de bateaux de guerre, 20 sortes de bateaux de course sont mentionnées; on y précise également les 37 façons de manier les avirons et les 37 chants et musiques de bateaux. Cette classification des bateaux d'apparat et de propriétés des rois suffit à montrer leur importance symbolique et militaire, mais surtout la nécessité de contrôler leur développement.

51 Les bateaux de cérémonie transportent les gens importants (bateau du roi, bateau des ministres, bateau des officiers, bateau des musiciens, etc.); ils portent des pavillons royaux, des temples, etc. Ils sont chargés de faire reconnaître par les hommes la majesté du pouvoir qui défile et se présente comme le gardien de l'ordre cosmique et social. 


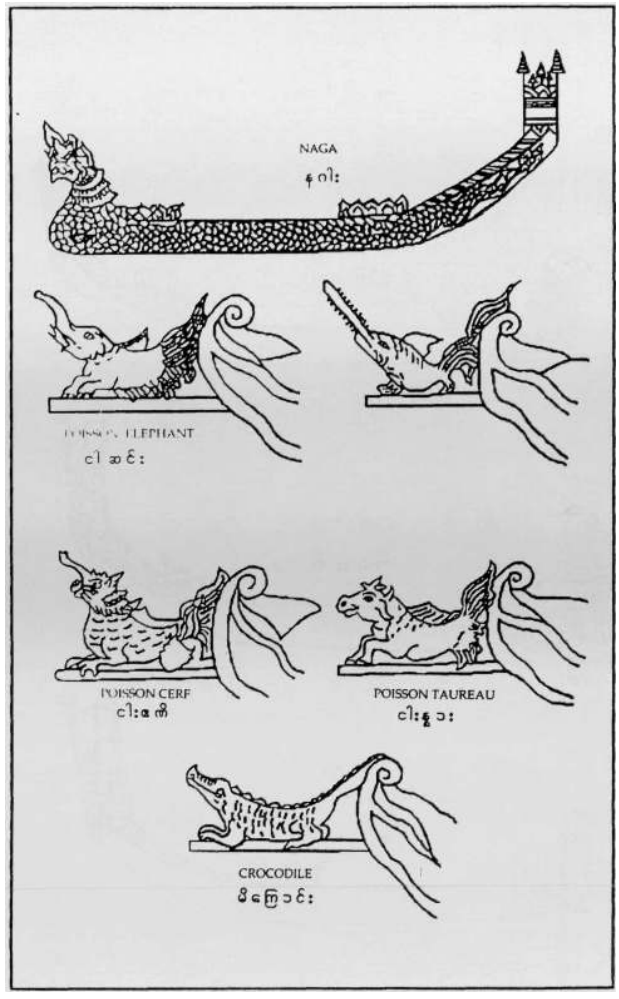

Figure 16. Différentes figures de proue (Robinne 1995 : 56-57)

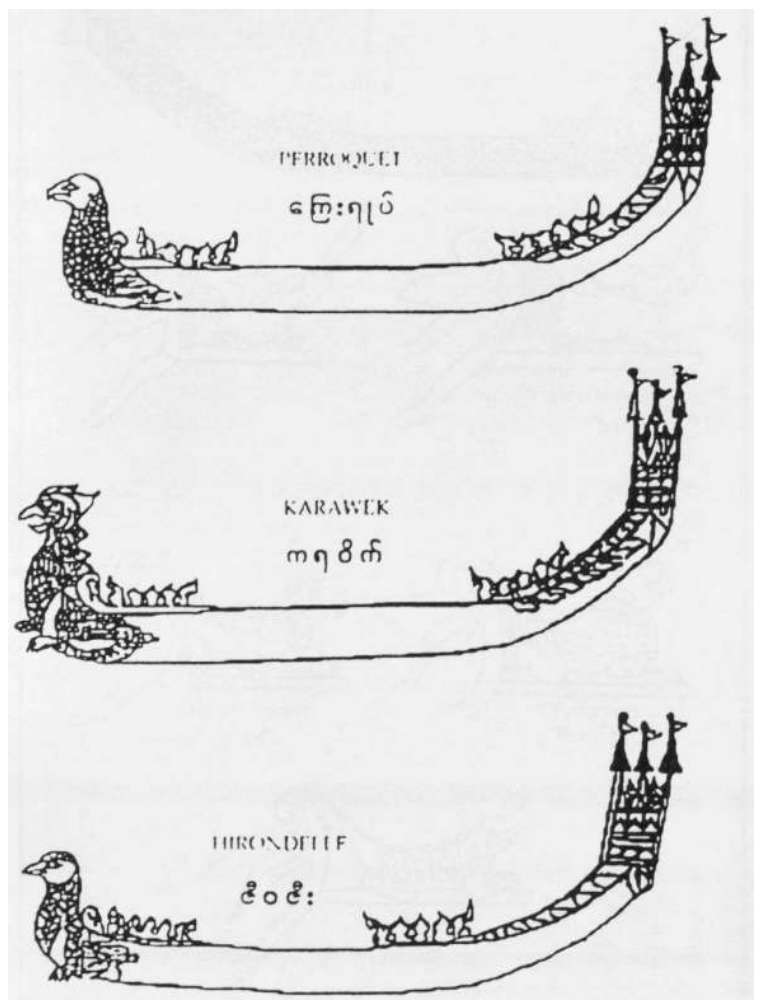

Figure 17. L'importance des oiseaux dans la symbolique des proues (Robinne 1995 : 58-59)

Enfin, chaque bateau royal a sa légende et son histoire, particulièrement les monoxyles. Puisant leurs origines dans les fûts des arbres cosmiques et originaux du centre du 
monde par exemple, ils sont individualisés, personnalisés, et sont devenus les symboles d'un monde supérieur ${ }^{22}$.

Rapportons et résumons ici la légende "officielle» des bateaux des rois birmans et notamment celle des quatre bateaux. A l'époque des rois Thaton, il existait un énorme arbre, appelé Peinne $\mathrm{Ma}$, dont les quatre branches pointaient dans chaque direction cardinale. Sur la première, se trouvait une tour d'où sortaient des voix charmantes ; sur la seconde, un écureuil volant; sut la troisième, un reptile; et sur la quatrième, une flèche de pagode. Les brahmanes conseillèrent au roi de transformer cet arbre en quatre bateaux.

54 Le bateau issu de la première branche fut appelé Thali $\mathrm{Ka}$ (la tour) car il s'agissait pour les brahmanes d'un avatar de Sakka, le dieu du tonnerre, représenté sur la proue versant de l'eau lustrale d'une conche (fig. 18).

Le bateau issu de la deuxième branche fut appelé Kama Kaw parce qu'il fut réalisé par les habitants du pays des Môns et représentait une créature avec une face de tigre. Une autre version rapporte qu'un poisson vivait sut cette branche (fig. 19).

Le bateau issu de la troisième branche fut appelé par les Môns - qui le construisirent Ah Kin, du nom d'un reptile (fig. 20).

Le bateau issu de la quatrième branche fut appelé Thara Beik Man parce qu'il ressemblait à une flèche de temple (fig. 21).

Le tronc de cet arbre gigantesque devint un bateau royal et fut appelé plus tard Min See Hlay.

À l'origine - et tout au long de l'histoire des rois birmans-, les navires de prestige et de cérémonie étaient faits à partir d'une coque monoxyle, celle-ci puisant ses racines dans le monde des mythes et de la religion. Ils sont donc chargés de puissance puisqu'ils permettent à l'identité et à la sacralité d'un peuple de s'exprimer, alors que le bateau en planches n'est plus qu'un instrument économique, dénué de l'essentiel de ces symboles, coupant le peuple de ses racines. C'est pourquoi le bateau monoxyle loñ hlè est si important et n'a pu (du moins pas encore) être détrôné par les bateaux aux coques et bordés en planches. 


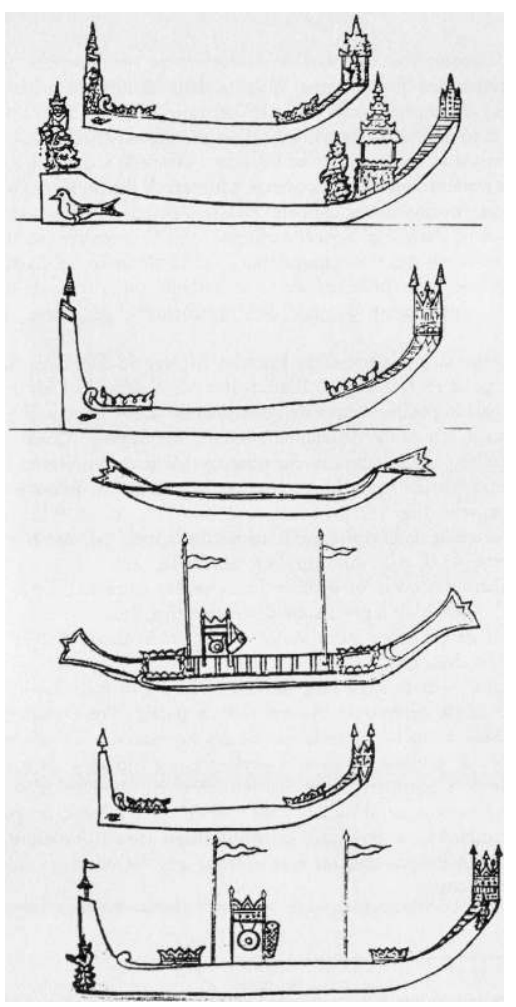

Figures 18-19-20-21. Les bateaux légendaires

\section{Offrandes et rituels}

Certains sages et des anciens, versés dans la tradition, savent renouer avec les fils du passé, bien que de plus en plus, les systèmes dominants et modernes (idéologie nationale, « productivisme » et parfois protectionnisme exacerbé) s'imposent, non sans arrogance, aux traditions. Les techniques et les rituels s'adaptent généralement facilement aux contingences des dominants; le bouddhisme lui-même a intégré (et parfois désintégré) ou protégé les croyances traditionnelles. Les maquettes monoxyles suspendues au-dessus d'autels "populaires » ajourés, que l'on peut observer à Mergui, sont placées en contrebas des pagodes comme pour bénéficier de leur protection; on en trouve aussi dans les autels réservés aux nats sur les plages des îles. On remarquera enfin que les bassins de refroidissement des forgerons ont la forme d'un bateau monoxyle -symbolisant peut-être le recours à un esprit d'eau douce- à la proue élancée comme un éperon, la poupe pouvant être droite.

61 Tous les marins honorent le nat situé à la proue du bateau avant chaque départ, et cela quel que soit le bateau ou la forme de sa coque. Ils lavent la «tête " de l'embarcation, prient mains jointes refermées sur des bougies, des bâtons d'encens, des fleurs, etc., offrandes ensuite déposées sur l'étrave. Qui est ce nat? Il s'agit de U Shingi ${ }^{23}$, localement reconnu comme "le nat de l'eau salée». Il ne fait pas partie des 37 nats officiels mais possède pourtant un culte reconnu, invoqué et respecté par tous les pêcheurs et même dans la plupart des foyers ; à l'origine, c'est le nat de l'eau saumâtre, le nat du delta, donc. Mais certains marins honorent aussi, et parfois surtout, Na Shema ${ }^{24}$, localement reconnu comme "le nat de l'eau douce ", situé aussi dans l'étrave (communication personnelle de B. Brac de La Perrière). 

deux nats, nous rappelle que le culte d'État s'oppose parfois aux cultes locaux et que ceux-ci répondent plus facilement à l'évolution technique. Ce n'est pas parce que les nats n'ont pas été intégrés par l'acte de reconnaissance officielle émis par la monarchie birmane qu'ils n'existent pas et ne sont pas invoqués. Il circule en effet des listes « complémentaires ", et les nats se transforment en fonction des besoins et des régions, avatars des nats plus « visibles ». U Shingi et Na Shema nous ramènent cependant aux rives de l'Irrawadi et dans les eaux du delta, d'où ils sont originaires; or, nous avons étudié l'influence technique de la batellerie sur les embarcations de mer; il y a donc évolution complémentaire du technique et du rituel. Ces deux nats se sont ensuite spécialisés pour répondre aux besoins spirituels des marins, l'un devenant le nat de l'eau salée, de la mer -masculin-, et l'autre celui de l'eau douce -féminin.

Certains informateurs nous ont déclaré que l'esprit du bateau se transmettait à l'intérieur d'une même lignée familiale ; il s'agit donc d'un esprit domestique dont nous avons parlé plus haut, emporté dans le bateau, renforçant l'équivalence entre bateau et maison, et expliquant la facilité avec laquelle les Birmans s'adaptent à une vie familiale semi-nomade sur les bateaux.

Sur certains bateaux réside donc un autre esprit que celui de l'étrave. Nous le définissons comme «structurel » puisqu'il est nécessaire à la construction d'un bateau loñ hlè. Il se trouve dans les varangues de fond de cale (fig. 22). Les varangues du bateau qui accueillent un nat sont des tégugwé (ou tegugwé, tagugwé), un terme qui vient de tam, désignant un objet allongé (un manche par exemple), de ku, un support et de gwé, une arche. Elles peuvent être constituées de deux pièces encastrées (fig. 23). Les termes techniques de la construction navale sont donc induits par leur fonction, les bateaux eux-mêmes étant désignés en fonction de leur forme.

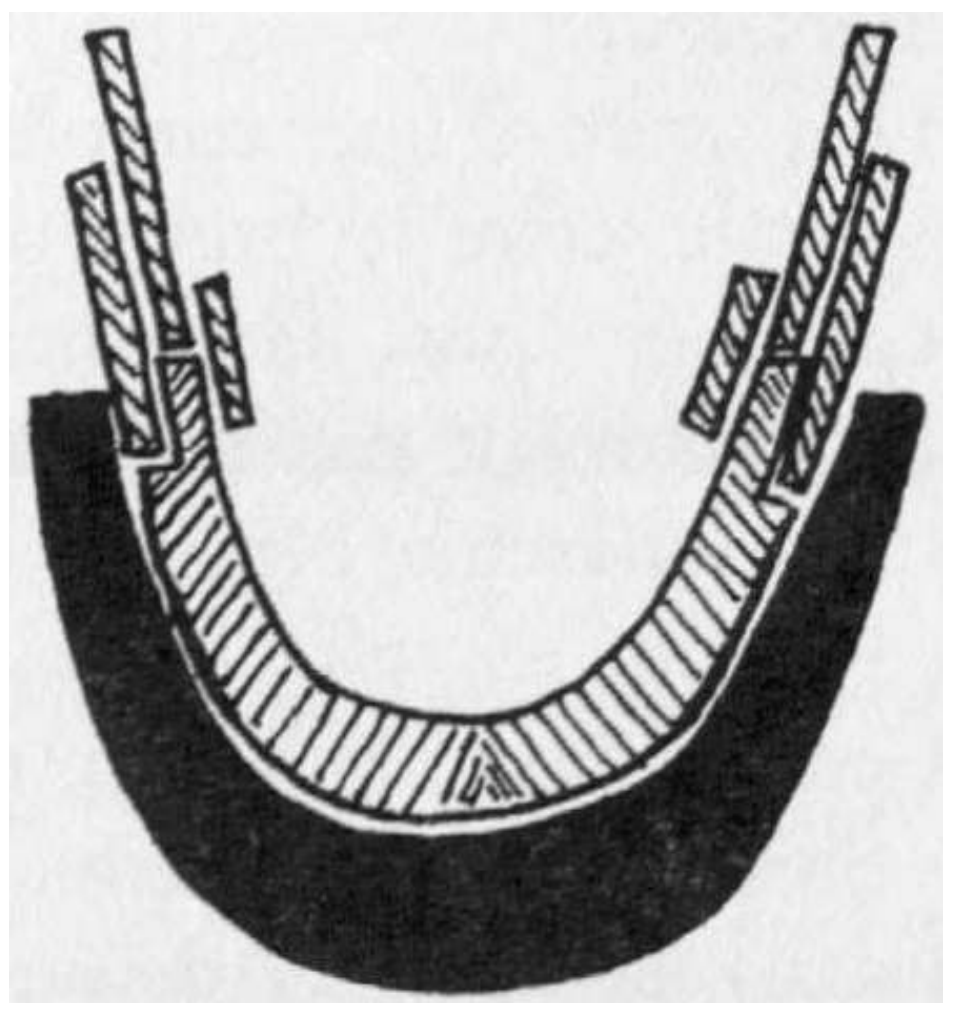

Figure 22. Membrures et varangues solidaires (L. Gansser) 


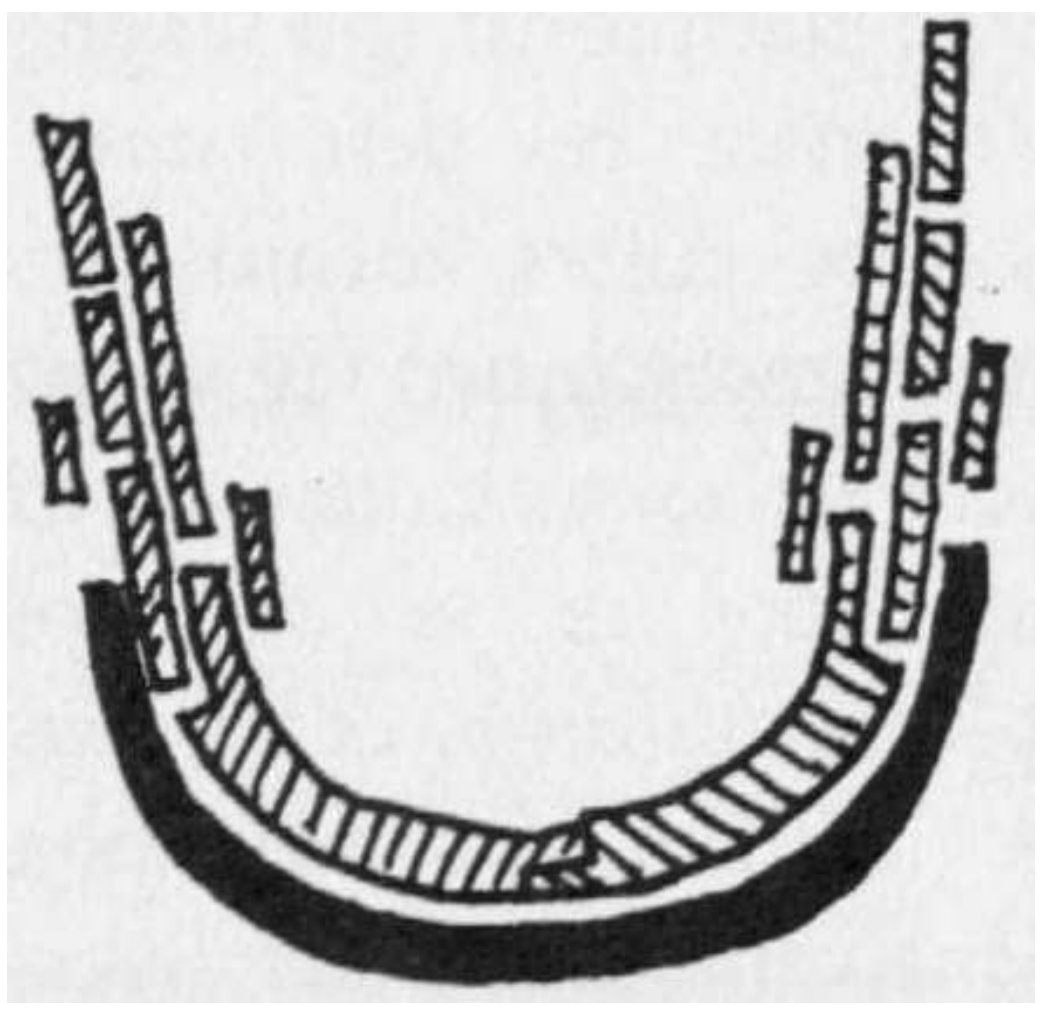

Figure 23. Membrures et varangues faites de 2 pièces (L. Gansser)

différenciés localement selon leurs étraves, pièces maîtresses lors de la construction, et non plus seulement en fonction de la forme allongée ou plate. L'étrave, localement appelée yarzatai ${ }^{28}$ est un gabarit de bois épais et carré dont la longueur varie en fonction de la taille désirée du bateau. Dans cette étrave, droite, se trouve un nat, U Shingi le plus souvent ; dans ce cas il n'y a pas de nat dans les varangues. nat relie les deux bords grâce à une varangue taillée dans une seule pièce ; si elle est en planches, le nat se trouve dans l'étrave. Mais que ce soit une varangue ou une étrave, le nat s'installe toujours dans la pièce maîtresse, c'est-à-dire, pour les Birmans, celle qui relie les deux bordés de coque. L'esprit serait cependant différent dans les deux cas ; U Shingi, un esprit associé à la mer, est masculin pour l'étrave et $\mathrm{Na}$ Shema, un esprit plutôt associé aux eaux douces et féminin, pour le monoxyle. 
Des offrandes sont faites dans les chantiers navals avant chaque lancement et pour chaque étape importante de la construction. Des offrandes de noix de coco, de canne à sucre, de noix d'arec, de feuilles de bétel, de chaux, de tabac, de boissons non alcoolisées, de bougies, d'encens sont présentées aux esprits. Une cérémonie d'offrande au nat U Shingi dans un chantier naval, accomplie par un spécialiste, est appelée puzaw pwé. Dans certains chantiers de réparation ou d'entretien (situés à côté des maisons dont les pilotis baignent dans la mer à marée haute), les offrandes sont transportées de la maison au bateau, le plus souvent dans le bol réservé aux bonzes pour leur quête matinale de nourriture. Elles sont ensuite placées sur la proue du monoxyle où l'évidage démarre, c'est-à-dire à l'endroit où les deux bordés se rejoignent. Là encore, la notion de lier et de rejoindre est importante. Les offrandes sont rapportées ensuite à la maison des donateurs; ce va-et-vient symbolise peut-être le transfert de l'esprit du foyer dans le bateau et le retour de l'âme de la famille à la maison. Il s'agit à nouveau d'une dialectique binaire terre/mer, foyer/bateau, qui rappelle la fonction symbolique du tégugwé.

\section{Conclusion : évolution technique et rituelle}

La construction des loñ hlè parvient à un équilibre parfait entre taille et élévation des bordés sur quille monoxyle, ce qui répond aux besoins socio-économiques d'un peuple de plaines et de vallées parti à la conquête de ses eaux.

70 Les pirogues monoxyles de rivière copiant le modèle de la pirogue de course se sont agrandies et leurs proues se sont creusées peu à peu en forme d'échancrure pour permettre, d'abord de manier la perche de propulsion, puis de détourner le bateau des rochers et autres dangers flottants. Les pirogues de course se sont développées avant toutes les autres embarcations car elles bénéficiaient du soutien de l'administration de l'État et de la religion; leur aspect esthétique a dominé le développement technique, pris en charge par les marins, pêcheurs, transporteurs ou marchands. Les décorations (yeux), les rituels (pour favoriser la mousson), la symbolique (image du dragon) et l'élancement de la poupe (queue de naga ou gravures sur bois par exemple) ont d'abord transformé ces pirogues en embarcations rapides.

71 Le transport fluvial se développant avec le pouvoir central et les échanges, les chargements devinrent de plus en plus importants (fig. 24). Pour mieux observer les eaux, les charpentiers ont surélevé la poupe, ce qui permit l'installation d'un gouvernail axial. Cette surélévation s'est inspirée des modèles de jonques chinoises mais aussi des modèles de pirogues rituelles et des bateaux royaux dont ils ont gardé la symbolique. Les animaux fabuleux de la mythologie indienne, aux avatars multiples, notamment l'oiseau, demeurent les modèles dont s'inspirent la technique, l'esthétique et le symbolique. Ces bateaux rappellent les montures des dieux de l'Inde et la proue des barques royales se confond avec une entrée de pagode protégée, de chaque côté de la rampe d'accès, par des animaux issus de la forêt Himaphan, celle qui borde le mont Méru, le centre de la cosmologie bouddhique.

72 Puis la descente des Birmans vers le sud a favorisé une navigation littorale et interîlienne (nous ne parlons pas ici des gros bateaux de transport et autres jonques), et le loñ hlè est apparu, supplantant des bateaux peu performants dont on trouve des vestiges avec les embarcations secondaires (fig. 25). Tentons une hypothèse quant à 
l'origine et à l'évolution des loñ hlè birmans dont les principales étapes seraient les suivantes :

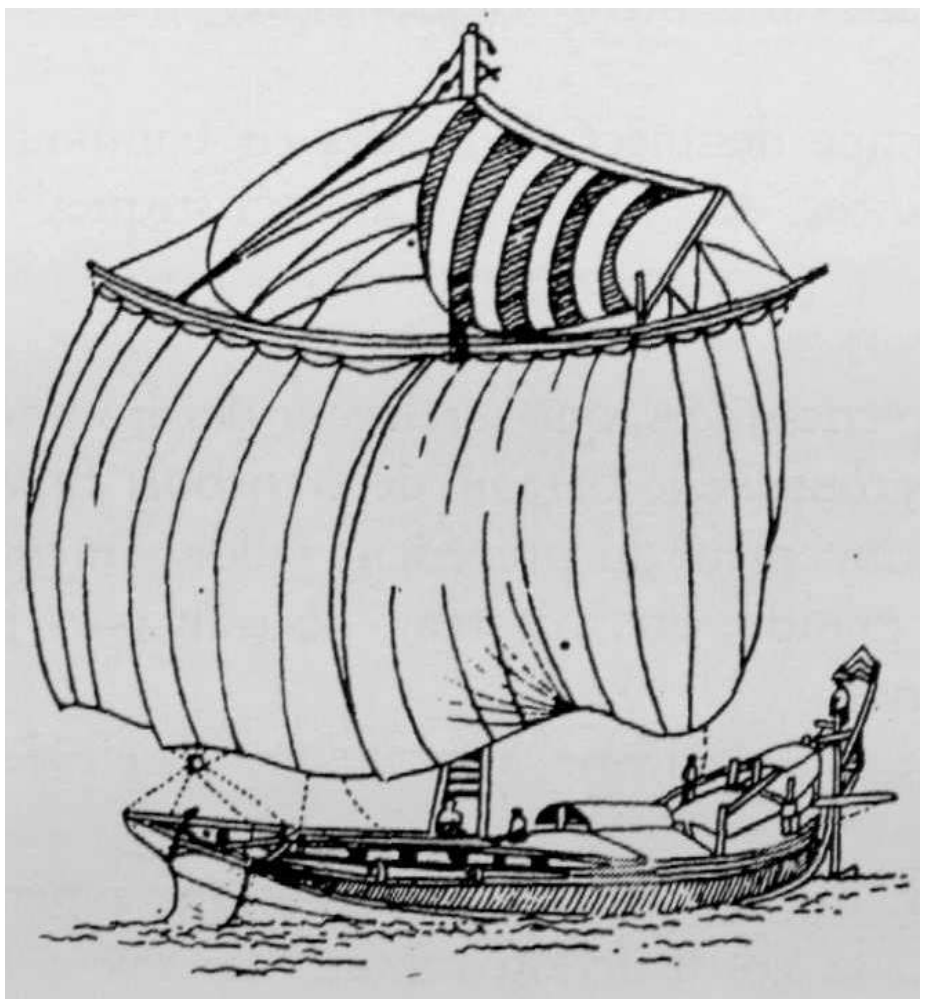

Figure 24. Un taba (Myanmar-english Dictionnary 1998: 162)

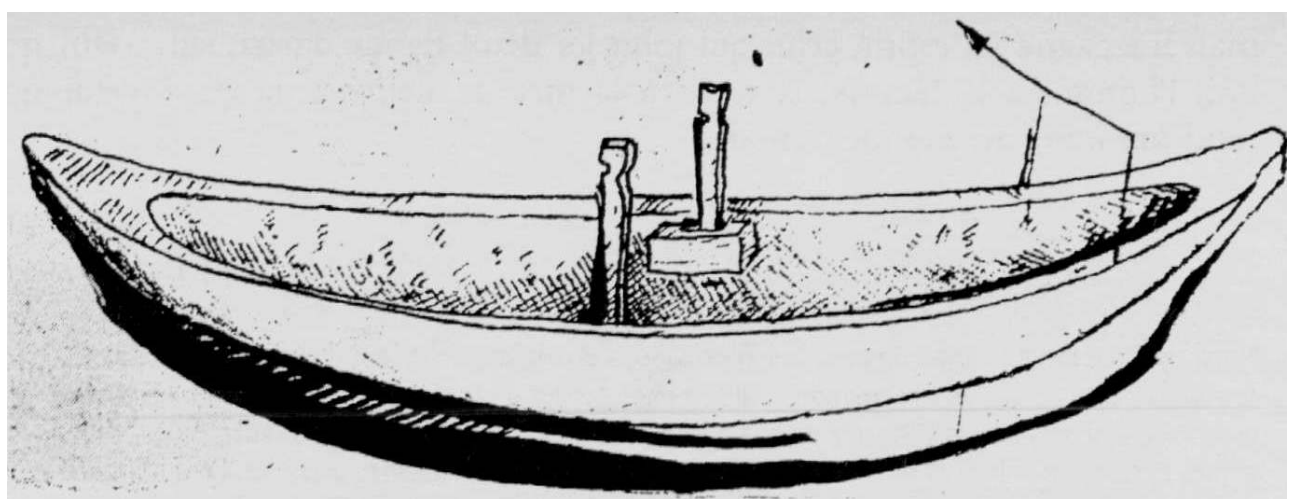

Figure 25. Les embarcations secondaires sont des témoins de l'existence d'anciennes pirogues monoxyles ; ici une pirogue avec monoxyle affiné dans sa partie supérieure, faisant office de bordé et assurant une stabilité minimale (L. Gansser)

1. le monoxyle simple, sans pavois ni bordé, ni proue ou poupe sculptée (embarcation pour les lacs, rivières et corvées ménagères ou petits travaux);

2. le monoxyle avec proue et poupe dessinées selon la « mode birmane », semi-échancré (embarcation pour la pratique d'une petite pêche ou pour le transport fluvial) ;

3. le monoxyle avec proue et poupe dessinées que surélève un pavois sans varangues (début de spécialisation des groupes de pêcheurs fluviaux, et fonction de protection);

76 4. le monoxyle avec proue et poupe dessinées et pavois avec varangues (spécialisation, pêcheurs installés dans une niche économique, mais aussi pavois de protection) ; 
77 5. le monoxyle avec proue et poupe dessinées et bordés en bambou ou rondins de bois rappelant l'intégration des flotteurs caractéristiques des pirogues indonésiennes (bateau de transport et premier développement du bordé de coque);

6. le monoxyle avec proue et poupe dessinées, que surélèvent des bordés en planches, loñ ko, qui a donné deux groupes : le bateau de transport fluvial, cargo, pin ko; et le loñ cap, un développement du précédent (allongement de la poupe et de la coque, voilure plus grande, etc.). bateau de transport plus important et pouvant affronter les mers ;

7. le loñ hlè, petit bateau de pêche maritime avec poupe surélevée et monoxyle non échancré.

La non-spécialisation des bateaux, ou leur faible spécialisation, a permis une adaptation rapide et simple de la construction navale. Le loñ hlè a perdu son échancrure, conservant sa poupe mythique et royale qui permet aux charpentiers de marine de s'exprimer et aux croyances de survivre; mais il a gagné un esprit, celui qui joint les deux flancs du bateau, celui qui unit l'homme à la femme, la terre à la mer, le fleuve à la mer, celui qui rend son unité au monde birman.

\section{BIBLIOGRAPHIE}

Autour du monde. Aquarelles. Souvenirs. Voyages

s.d., Fascicule XII, Birmanie. Sites et Paysages. D'après les clichés photographiques de MM. Bourne et Sheperd, Paris. 8 planches couleur +2 pages de texte.

Bernot, Denise, 1979-92, Dictionnaire franco-birman. Langues et Civilisations de l'Asie du Sud-Est et du Monde Insulindien. Paris : Éditions Peeters, Selaf (14 vol.).

Brac de La Perrière, Bénédicte, 1989, Les rituels de possession en Birmanie. Du culte d'État aux cérémonies privées. Paris : Éditions Recherche sur les Civilisations.

Charney Michael W., 1997, « Shallow-draft Boats, Guns, and the Aye-ra-wa-ti. Continuity and Change in Ship Structure and River Warfare In Precolonial Myanma », Oriens Extremus 40, 1 : 16-63.

Condominas, Georges, 1986, « Ritual Technology in Mnong Gar Swidden Agriculture », pp. 28-46, in I. Nørlund, S. Cederroth, I. Gerdin (eds), Rice Societies. Asian Problems and Prospects. Copenhague : Scandinavian Institute of Asian Studies («Studies on Asian Topics » 10).

Ferrars, Max. and Bertha, 1996, Burma. Bangkok : Ava Publishing House (1ère édition 1901, Sampson Low, Martson \& Co. Ltd, London),

Forrest, Thomas, 1792, A Voyage from Calcutta to the Mergui Archipelago, Lying on the East Side of the Bay of Bengal. London : Robson.

Holbrock, R. D. et Manoh Suriya, 2000, Blue Book of Coastal Vessels. Bangkok : White Lotus Press (bilingue thaï et anglais) (1ère édition, Joint Thai-US Military Research and Development Center, 1967). 
Ivanoff, Jacques, 1999, « L'esclavage ou le prix de la liberté nomade », pp. 41-94, in Formes extrêmes de dépendance. Contribution à l'étude de l'esclavage en Asie du Sud-Est. Paris : EHESS («Civilisations et sociétés »).

- 1999, The Moken boat : Symbolic technology. Bangkok : White Lotus.

Judson's Burmese-English Dictionnary, 1953, Rangoon, Baptist Board of Publications, Centenary Edition.

Myanmar-English Dictionnary, 1993, Department of the Myanmar Language Commission, Ministry of Education, Union of Myanmar (réed. 1998).

Manguin, Pierre-Yves, 1993, « The Vanishing Jong : Insular Southeast Fleets in Trade and War. Fifteenth to Seventeenth Centuries ", in A. Reid (ed.), Southeast Asia in the Early Modern Era : Trade, Power, and belief. Ithaca/New York : Cornell University Press.

Pe Maung Tin (U) et Gordon H. Luce, 1960, The Glass Palace Chronicle of the Kings of Burma. Rangoon : Rangoon University Press (1ère édition 1923).

Poujade, Jean, 1946, La Route des Indes et ses navires. Paris : Payot.

Robinne, François, 1990, « Le littoral oublié des Birmans », ASEMI (Asie du Sud-Est et Monde Insulindien) XIV (3-4) : 181-184, « Rôles et représentations de la mer » (en collaboration avec le SERIA).

- 1995, « Mers mythiques d'Asie du Sud-Est continentale et représentations symboliques des figures de proue au Myanmar », CETMA- Anthropologie maritime 5 : 47-62.

- 1995, « Pays de mer et gens de terre : logique sociale de la sous-exploitation du littoral en Asie du Sud-Est continentale », Bulletin de l'Ecole Française d'Extrême-Orient : 181-216.

Singer, Noel F., 1993, Burmah. A Photographic Journey, 1855-1925, Paul Strachan Kiscadale.

Slee, David, n. d., , « Longtails. Évolution to Revolution ? », The Greater Phuket Magazine 11, 4 : $20-30$ et 82 .

Thierry, Solange, 1985, Le Cambodge des contes. Paris : L'Harmattan.

Yule, Henri, 1860, « Voyage dans le royaume d'Ava (empire des Birmans), 1855 », Le Tour du Monde, tome II.

\section{NOTES}

2. Pourtant des Karens et des Môns ont exploité le littoral depuis des temps très reculés mais n'ont jamais été assez nombreux pour exploiter l'immense archipel. Les Môns ont cependant certainement donné aux proues birmanes leurs légendaires origines.

3. La culture birmane, comme d'autres en Asie du Sud-Est continentale, repose sur le travail de la terre, et particulièrement sur la riziculture. Elle seule donnait statut et pouvoir.

4. Les freins à l'innovation sont le fait des autorités qui ne veulent pas permettre une trop grande ouverture économique et, de là, l'émergence d'une classe moyenne dangereuse pour le pouvoir.

5. On se reportera à la réédition de l'ouvrage de R. D. Holbrock et Manob Suriya (2000:261-267) qui mentionne la présence de ces bateaux dans les eaux de Ranong, en Thaïlande, ville frontalière avec la Birmanie.

6. Les routes maritimes assuraient un réseau de communications entre le sous-continent indien et l'Asie du Sud-Est, et les relations maritimes existaient depuis les temps préhistoriques. Pour rejoindre les royaumes "intérieurs» les marchands, militaires et religieux empruntaient principalement deux routes : la première allait de Takuapa ou du Kedah à travers l'isthme de Kra 
pour rejoindre Chaiva ; l'autre, la fameuse route des Trois Pagodes, allait de Tavoy pour rejoindre les vallées de la rivière Menam au Siam, la voie de communication la plus importante de ce pays.

7. Pour F. Robinne, le terme hlè désigne tous les bateaux. À ses yeux, le terme loñ désigne, à l'origine, une pirogue monoxyle mot qui peut être d'origine mône, dlun, et mentionné dans les inscriptions (Pe Maung Tin et Luce 1960: 327 et 346). Il s'agit en fait d'une pirogue de forme allongée, à l'origine taillée dans une masse, mais pas forcément monoxyle. Les informateurs transcrivent long hlay (que nous avons simplifié en loñ hlè).

8. Il s'agit du loñ zat (ou sat ou encore cap terme désignant à l'origine un bateau monoxyle avec des bordés en planche)

9. M. et B. Ferrars (1996) rapportent des laung-ngô ou laung-go que F. Robinne (1995 : 33) appelle pin:ko. Pour cet auteur, c'est une embarcation à proue convexe, au bordé doublé de bambou flotteur qui serait d'origine shan. Il remarque que les loñ (qu'il appelle les luan:) sont à proue concave et se nommeraient laun : ko dans leur version transport de marchandises.

10. En fait mut ka tu (ou mo'gedou) : «The short thick board placed at the bow and stern of a Burmese boat, in which the gunwales are inserted.» (Judson 1953).

11. Les Birmans ont donc choisi des termes de marine découlant de la fonction technique des pièces de charpenterie plutôt que de leur donner des symboles anthropomorphes.

12. Les flotteurs sont aussi une tradition austronésienne et surtout océanienne. Les premières embarcations des villages littoraux, lacustres (pour collecter les légumes et les autres produits de la forêt ou de la rivière) étaient des petits monoxyles, en fait de simples flotteurs évidés, parfois liés ensemble (Autour du Monde, fascicule XII, cliché XCV) par des traverses formant ainsi les premières pirogues à balancier. On trouve des pirogues surélevées d'un pavois/coffrage à Madura (Poujade 1946 : 281) auquel s'est ajouté un flotteur précurseur du balancier. Plus on descend vers le monde océanien, plus les flotteurs se désolidarisent de la coque alors que, plus on remonte vers l'Asie continentale, plus les flotteurs collent à la coque.

13. Sur l'île d'Aleman par exemple où même les kabang moken, ont été remplacés par des bateaux de facture birmane sous la très forte pression des autorités.

14. Pour les jonques et leur disparition (aux alentours du XVI ${ }^{\mathrm{e}}$ siècle), voir P.-Y. Manguin (1993), et J. Poujade (1946: 243, note 2) : «On constate dans le golfe du Siam, par exemple, que certaines jonques armées par les Chinois ou des métis de Chinois ont des voiles lattées mais des coques symétriques. Comme le marin qualifie le plus souvent un bateau d'après la voilure et le gréement, plus visibles à la mer que la coque, on appelle ces bateaux des jonques. En réalité elles ont des formes qui n'ont rien de chinoises. »

15. On trouve ce nom un peu partout en Asie du Sud-Est. Il s'agit d'un bateau de transport chinois à l'origine, copiant ses lignes sur le modèle «canard» des Chinois (Poujade 1946 : 210-211). Les sampans sont devenus très courants dans les eaux birmanes ou thaillandaises et ont été adaptés par les charpentiers en fonction des demandes locales, prenant des formes et des noms variés. En Thaillande, on le trouve sous des noms divers (sampan chang, sampan suan, etc.) et les jonques se sont développées sous le règne de Rama II. On trouve en outre un ma pet, «bateau canard » en Thaillande, modèle courant de transport en eau douce, ce qui, à nouveau, nous rapproche de la Chine.

16. La Thaïlande a aussi construit des pirogues monoxyles pour naviguer sur ses fleuves et son littoral (rua maht, rua chala, rua $\mathrm{mu}$ ) et les premiers modèles, aux poupes et proues identiques surmontées d'un pavois, généraux en Asie du Sud-Est, sont semblables aux modèles birmans les plus simples. Mais aujourd'hui, plus aucun monoxyle thaillandais ne navigue sur les mers (à l'exception de ceux des Moken et des Malais). Ils ont été remplacés, depuis une cinquantaine d'années, par les long tails, autrefois équipés d'une voile latine, d'influence plutôt arabe (modelés à partir des dhow arabes qui ont donné les bateaux thaïs rua chalorm) que chinoise.

17. On consultera $\mathrm{H}$, Yule (1860: 2161-262) pour plus de renseignements. L'auteur a relevé une verge de $39 \mathrm{~m}$ de haut et plus de $360 \mathrm{~m}^{2}$ de voilure. 
18. Il s'agit de sculptures florales sur bois (très fréquentes dans les décorations des pagodes) inspirées du kanou, «traditional art style of depicting convoluted lotus stems, buds, blossoms, etc. " (Myanmar-English Dictionnary 1998 : 3), et issus des entrelacs décoratifs du siège du barreur du bateau loñ ko (cf. par exemple Autour du Monde, fascicule XII, planche XC1I ou N. F. Singer 1993 : 74).

19. On retrouve ce principe sur les pirogues cambodgiennes. «Un mât planté devant la cabane porte une voile aurique, et trois hommes manœuvrent chacun un aviron, estropé à des tolets placés deux en avant et un en arrière. L'homme de barre manœuvre le dernier aviron en gouvernant avec sa jambe.» (Poujade 1946 : 213).

20. L'existence des oiseaux psychopompes est générale en Asie du Sud-Est (tourterelles des monuments funéraires cambodgiens, oiseaux assistants du chamane, autres tourterelles sacrées des Moklen par exemple). Les oiseaux sont aussi symboles du pouvoir et de la royauté (garuda de la Thaïlande), etc., et selon certaines traditions (rapportées par F. Robinne 1995), ces oiseaux mythiques permirent au Bouddha lui-même de rejoindre l'Asie du Sud-Est à partir de l'Inde et/ou de Ceylan. D'autres animaux imaginaires sont présents dans ces deux dominant les eaux de l'Océan Indien, des oiseaux mécaniques par exemple, précurseurs de nos robots (Thierry 1985).

21. Le htee est l'ornement en fer qui couronne le dôme de toutes les pagodes.

22. Il n'est donc pas surprenant de trouver des bateaux ayant un rôle psychopompe essentiel; d'une manière plus générale, en Asie du Sud-Est, le bateau est le moyen utilisé par le Bouddha pour traverser les eaux tumultueuses des réincarnations et de la vie. Le bateau mène au paradis, c'est le véhicule des dieux.

23. Localement appelé, entre autres, yengipai-u-shin-gyi, «le nat de l'eau salée » selon la transcription et la traduction de nos informateurs; il s'agit en fait de $u$ bran kri, "monsieur seigneur grand $»$.

24. En fait nat bram ma, « nat seigneur féminin », qui ne possède pas de forme bien définie et dont le culte est plus difficile à définir.

25. En tout cas, certaines d'entre elles, posées au fond du maître-bau.

26. Un pwé est une cérémonie aux nats au cours de laquelle on assiste à un spectacle, à des offrandes, à des possessions et à la mise en circulation de biens et d'argent...

27. B. Brac de La Perrière rapporte l'existence d'esprits féminins dans les barrots/bancs de nage de certaines embarcations fluviales (communication personnelle).

28. Il s'agit en fait d'un mot dérivé de celui utilisé par les Birmans : jaza dan (« roi, pièce verticale »).

\section{RÉSUMÉS}

Les loñ hlè, bateaux de pêche monoxyles du Ténasserim, dominent Les eaux de l'archipel Mergui sillonnées par les nomades de la mer moken, les marins karens et birmans. Ces bateaux mesurent entre 8 et 24 mètres, et sont taillés dans les grands fûts d'Hopea, de Shorea et même de teck. Leurs élégantes poupes élancées, leurs proues droites, concaves ou échancrées, Leurs pavois autrefois, puis leurs bordés en planches, sont leurs principales caractéristiques. Les origines du loñ hlè seront définies, leurs grands ancêtres demeurant les pirogues rituelles et de course. Chaque embarcation possède son esprit, nat, situé sur l'étrave. Mais d'autres nats vivent sur le bateau, et d'autres encore rappellent la relation entre le bateau et la maison. Enfin ces nats 
forment souvent un binôme fonctionnel pour l'embarcation et sont associés à l'eau salée et à l'eau douce, une ambivalence qui rappelle l'évolution historique de la technologie navale birmane.

The loñ hle, monoxyla fishing boats of the Tenasserim are found all over the seas of the Mergui Archipelago furrowed by the Moken sea nomads, the Karen and Burmese seamen. They are between 8 and 24 meters long and are carved out of the large boles of the hopea odorata, heritiera javanica and teak trees. Their elegant tapered sterns, vertical, concave or notched, prows, their former small bulwarks and the plankings of their hulls which are used today with three planks are the most remarkable characteristics of such boats. The origin of the loñ hlè is an avatar for the sea of the pin ko, a river boat. The great ancestors remain the ritual pirogues and those used for races. Each boat possesses its own spirit, nat, situated in the stern of the boat, most often $U$ Shingi. But there are other nats that live on the boat, in other symbolic technically strategic parts, notably in the ribs; other nats (and even sometimes the same ones) are transmitted from one generation to another of the same family; they are the domestic spirits that recall the relation the boat has to the home. Finally, these nats often have a two fold function in the boat and are associated to salted water and fresh water, an ambivalence that reminds one of the Burmese historical and naval evolution.

Los loñ hlè, barcos de pesca monoxilos de Tenasserim, dominan las aguas de Tenasserim, dominan las aguas des archipiélago Mergui recorridas por los nómades del mar moken, los marinos karens y birmanos. Estos barcos miden entre 8 y 24 metros y son tallados en grandes troncos de diversos árboles. Sus elegantes popas esbeltas, sus proas derechas, cóncavas o talladas, sus pabellones en otros tiempos, y sus bordos de tablas de madera son sus principales características. Los orígenes del loñ hlè serán definidos, sus grandes ancestros son siempre las piraguas rituales y de carreras. Cada embarcación posee su espíritu, nat, situado sobre la traba. Pero otros nats viven sobre el barco y otros, además, recuerdan la relación entre el barco y la casa. En fin esos nats forman frecuentemente un binomio funcional para la embarcación y están asociados al agua salada y al agua dulce, resultando une ambivalencia que recuerda la evolución histórica de la tecnología naval birmana.

INDEX

Mots-clés : Birmanie, esprit domestique, littoral, Mergui, monoxyle, nat, rituel, Ténasserim

\section{AUTEURS}

\section{LUCA GANSSER}

Artiste-peintre suisse, membre du du Mergui Archipelago Project, projet interdisciplinaire travaillant dans les eaux de l'archipel Mergui depuis 1998

\section{JACQUES IVANOFF}

Membre du MAP et chercheur au CNRS, « Techniques et culture », France 